\title{
Observer-Based Fuzzy Integral Sliding Mode Control For Nonlinear Descriptor Systems
}

\author{
Jinghao Li, Qingling Zhang, Xing-Gang Yan, and Sarah K. Spurgeon, Senior Member, IEEE
}

\begin{abstract}
This paper investigates observer-based stabilization for nonlinear descriptor systems using a fuzzy integral sliding mode control approach. Observer-based integral sliding mode control strategies for the T-S fuzzy descriptor systems are developed. A two step design is first developed to obtain the observer gains and coefficients in the switching function using linear matrix inequalities, and the results are used to facilitate the development of a single step design approach, which is seen to be convenient but introduces some conservatism in the design. The potential application to a class of mechanical systems is also considered. Since the descriptor system representation of mechanical systems is adopted, it is shown that in contrast to the existing fuzzy sliding mode control methods based on the normal system representation, the resulting T-S fuzzy system does not contain different input matrices for each local subsystem and the required number of fuzzy rules is consequently markedly reduced. Finally, the balancing problem of a pendulum on a car is numerically simulated to demonstrate the effectiveness of the proposed method.
\end{abstract}

Index Terms-Observer-based stabilization, T-S fuzzy descriptor systems, integral sliding mode control, mechanical systems.

\section{INTRODUCTION}

The Takagi-Sugeno (T-S) fuzzy model [1] has been extensively utilized as a popular and convenient tool to deal with complex nonlinear systems. With the T-S fuzzy model, nonlinear systems with smooth nonlinearities can be exactly represented in a compact set of the state space by a set of linear subsystems connected by corresponding normalized weighted coefficients. Then approaches to systematic analysis and synthesis for the resulting T-S fuzzy systems can be developed within the frame of conventional control technology and fuzzy logic control. As a result, this T-S fuzzy approach has attracted significant attention from the control community [2]-[12]. Despite the superiority of the T-S fuzzy model, the fuzzy rules will exponentially increase with the nonlinearities arising in the system representation. This increase in the number of fuzzy rules can produce a linear matrix inequality (LMI) condition which is infeasible or unnecessarily complicated. Recently,

This work was supported by the National Natural Science Foundation of China under Grant 61273008 and 61673099.

J. Li and Q. Zhang are with the Institute of Systems Science, State Key Laboratory of Synthetical Automation for Process Industries, Northeastern University, Shenyang 110819, China (e-mail: lijinghao@stumail.neu.edu.cn; qlzhang@mail.neu.edu.cn).

X.-G. Yan is with the Instrumentation, Control and Embedded Systems Research Group, School of Engineering and Digital Arts, University of Kent, Canterbury, Kent CT2 7NT, United Kingdom (e-mail: x.yan@kent.ac.uk).

S. K. Spurgeon is with the Department of Electronic and Electrical Engineering, University College London, Torrington Place, London, WC1E 7JE, United Kingdom (e-mail: s.spurgeon@ucl.ac.uk).

Corresponding Author: Q. Zhang (e-mail: qlzhang@mail.neu.edu.cn).
[13] presented a T-S fuzzy descriptor model which can be regarded as a generalization of the T-S fuzzy normal model $(E=I)$. The advantages of the T-S fuzzy descriptor model are a reduction in the number of fuzzy rules and a tighter nonlinear system representation [14]. Over recent years, a great deal of effort has been devoted to the study of T-S fuzzy descriptor systems and many significant results have been achieved in diverse areas, for example, stability and stabilization [15]-[23], observer-based control [24]-[25], guaranteed cost control [26][27], $H_{\infty}$ and dissipative control [28]-[31], and fault-tolerant control [32].

Sliding mode control (SMC) [33]-[36], as an effective robust control approach, has been widely applied to control and observe complex practical systems. The attractive features of SMC are its strong robustness properties and fast response. Recently, [7] gives a first attempt to remove the assumption that each local subsystem model shares the same input matrix in the sliding mode control problems for T-S fuzzy systems. Subsequently, by dividing the state space into several regions, the T-S fuzzy system is transformed into a linear uncertain system in each region. Then, using the sliding mode control method for each linear system and considering the continuity of the state trajectories over the region border, a piecewise integral sliding mode control for T-S fuzzy systems is presented in [8] to relieve the assumption pointed out in [7]. However, it is not a straightforward task to implement this sliding mode control strategy due to its high complexity. [11] formulates new states by incorporating the inputs and presents a dynamic integral sliding mode strategy to remove the assumption that each local subsystem shares the same input matrix for T-S fuzzy systems as pointed out in [7], but the dimension of the closed-loop system increases since the inputs are also a subset of the new states. Consideration of mechanical systems shows that they are generally in the form of nonlinear descriptor systems with a constant input matrix. The traditional approach [2], [7], [9], [35], [36] to the control of mechanical systems is to transform the system to an equivalent nonlinear normal system $(E=I)$ by performing the inverse operation of the derivative-term coefficient. This inverse operation will make the input matrix of the equivalent nonlinear normal system become nonlinear. When a T-S fuzzy model is used, the corresponding T-S fuzzy system will contain different input matrices for each local subsystem. A motivational example to illustrate this is the balancing problem of a pendulum on a cart in [7]. From this observation, it can be seen that the different input matrices of certain T-S fuzzy systems may not be an inherent system characteristic, but may be introduced artificially as a result of the modelling paradigm. In this paper, the 
first motivation is to study sliding mode control problems for mechanical systems in descriptor form. Mechanical systems are always described by T-S fuzzy descriptor systems where the derivative-term coefficients are different for each local subsystem [23]. In this case, the sliding mode control methods in [19], [31] for T-S fuzzy descriptor systems that share the same derivative-term coefficient for each local subsystem can not be directly applied. Moreover, the sliding mode control methods in [19], [31] are based on state feedback designs. In practice, it may be expensive or sometimes impossible to obtain precise measurements of all the system states. For the purpose of controller design, only the outputs are accessible [37]-[39]. In this case, an alternative approach is to use a state observer [40]-[41] to reconstruct the system states. It should be emphasized that when the T-S fuzzy descriptor systems contain parameter uncertainties, the separation principle usually does not hold [25], [32]. As a result, designing an observer-based sliding mode control strategy for T-S fuzzy descriptor systems with parameter uncertainties is the second motivation. To the best of the authors' knowledge, the observer-based sliding mode control problem for nonlinear descriptor system using a T-S fuzzy model has not been previously studied.

This paper is concerned with observer-based integral sliding mode control problems for nonlinear descriptor systems based on a T-S fuzzy model. Then an observer-based sliding mode control strategy is designed for the T-S fuzzy descriptor system. It is shown that the observer gains and coefficients in the switching function can be obtained by a proposed singlestep or two-step design approach, where although the singlestep design approach may introduce some conservatism in the design, it is more convenient than the two-step design approach. Moreover, the proposed methods in this paper are also utilized to control a class of mechanical systems. Since the nonlinear descriptor system is adopted to represent the mechanical system, the inverse operation of the derivativeterm coefficient of the nonlinear descriptor system is avoided. It is shown that, compared with the existing fuzzy sliding mode control methods [7], [9], [12], the resulting T-S fuzzy system does not contain different input matrices for each local subsystem and the required number of fuzzy rules is consequently markedly reduced. The contributions of this paper are threefold: 1) a systematic way to design an observerbased controller for a T-S fuzzy descriptor system with parameter uncertainties is presented via integral sliding modes; 2) considering that the separation principle is usually not satisfied for T-S fuzzy descriptor systems containing parameter uncertainties [25], [32], in this paper, a single-step approach and two-step approach are respectively proposed to evaluate the observer gains and coefficients in the switching function; 3 ) based on the proposed methods in this paper, an alternative way to control mechanical systems is presented. It is seen that with the descriptor representation, each subsystem of the resulting T-S fuzzy descriptor system shares the same input matrix and the artificial introduction of the different input matrices of each subsystem is avoided.

The paper is organized as follows: Section II defines the problem under consideration and gives some essential definitions and lemmas. Section III focuses on observer-based sliding mode control for T-S fuzzy descriptor systems. Section IV considers a class of mechanical systems and with the descriptor representation and the proposed methods in Section III, a new fuzzy integral sliding mode control method is presented for this class. Section V provides three examples to show the validity of the results proposed. Finally, Section VI concludes the paper.

Notation: The notation used throughout this paper is quite standard. $\mathbb{R}^{n}$ represents the $n$-dimensional Euclidean space, and $\mathbb{R}^{m \times n}$ represents the set of all $m \times n$ real matrices. The superscripts $T$ and -1 denote matrix transposition and matrix inverse respectively. $\mathbb{R}^{+}$represents the set of positive real numbers. $\|\cdot\|$ denotes the Euclidean norm of a vector or the induced norm of a matrix. The notation $P>0(P \geq 0)$ implies that $P$ is a real symmetric and positive definite (semipositive definite) matrix. $\mathbf{H e}(A)$ stands for $A+A^{T}$. The star $\star$ in a matrix block implies that it can be induced by symmetric position. Matrices, if their dimensions are not explicitly stated, are assumed to be compatible for algebraic operations.

\section{Problem Formulation AND PREliminaries}

Consider a class of uncertain nonlinear descriptor systems which can be represented by the following uncertain T-S fuzzy descriptor system

$$
\begin{gathered}
\sum_{k=1}^{r_{e}} v_{k}(z(t))\left(E_{k}+\Delta E\right) \dot{x}(t) \\
=\sum_{i=1}^{r} h_{i}(z(t))\left(A_{i}+\Delta A\right) x(t)+B u(t) \\
y(t)=C x(t)
\end{gathered}
$$

where $x(t) \in \mathbb{R}^{n}$ is the state vector, $u(t) \in \mathbb{R}^{m}$ is the control input vector, $y(t) \in \mathbb{R}^{l}$ is the measurable output vector. $z(t)=\left[z_{1}(t), z_{2}(t), \cdots, z_{p}(t)\right]$ is the premise vector and is measurable. $v_{k}(z(t)), k=1,2, \cdots, r_{e}$ and $h_{i}(z(t)), i=$ $1,2, \cdots, r$, are fuzzy membership functions in the left side and in the right-hand side, respectively. $E_{k}, k=1,2, \cdots, r_{e}$, and $A_{i}, B, C, i=1,2, \cdots, r$, are constant matrices of appropriate dimensions defining the $k$ th right-hand side subsystem and the $i$ th left-hand side subsystem of the T-S fuzzy descriptor system respectively. $\Delta E=M_{E} F_{E}(t) N_{E}$ and $\Delta A=M_{A} F_{A}(t) N_{A}$ with $F_{E}^{T}(t) F_{E}(t) \leq 0, F_{A}^{T}(t) F_{A}(t) \leq 0$ are parameter uncertainties. It is assumed that $\sum_{k=1}^{r_{e}} v_{k}(z(t))\left(E_{k}+\Delta E\right)$ is nonsingular.

Remark 1: The T-S fuzzy descriptor system (1) has a wide range of applications in the domain of mechanical systems and electro-mechanical systems [35]. A class of mechanical systems in descriptor form will be considered in Section IV to justify the potential applications of the T-S fuzzy descriptor system (1).

Define $\eta(t)=\left[\begin{array}{ll}x^{T}(t) & \dot{x}^{T}(t)\end{array}\right]^{T}$, the T-S fuzzy descriptor system (1) can be rewritten by its equivalent representation [14]

$$
\begin{aligned}
\mathbf{E} \dot{\eta}(t)= & \sum_{k=1}^{r_{e}} \sum_{i=1}^{r} v_{k}(z(t)) h_{i}(z(t))\left(\mathbf{A}_{k i}+\Delta \mathbf{A}\right) \eta(t) \\
& +\mathbf{B} u(t) \\
y(t)= & \mathbf{C} \eta(t)
\end{aligned}
$$


where $\mathbf{E}=\left[\begin{array}{ll}I & 0 \\ 0 & 0\end{array}\right], \mathbf{A}_{k i}=\left[\begin{array}{cc}0 & I \\ A_{i} & -E_{k}\end{array}\right], \mathbf{B}=$
$\underbrace{\left[\begin{array}{c}0 \\ B\end{array}\right], \mathbf{C}=\left[\begin{array}{ll}C & 0\end{array}\right], \Delta \mathbf{A}=\left[\begin{array}{cc}0 & 0 \\ \Delta A & -\Delta E\end{array}\right]=}_{\mathbf{M}} \underbrace{\left[\begin{array}{cc}\Delta A \\ M_{A} & -M_{E}\end{array}\right]}_{\mathbf{F}(t)} \underbrace{\left[\begin{array}{cc}F_{A}(t) & 0 \\ 0 & F_{E}(t)\end{array}\right]}_{\mathbf{N}}\left[\begin{array}{cc}N_{A} & 0 \\ 0 & N_{E}\end{array}\right]$.

Define sets $H=\{1,2, \cdots, r\}, V=\left\{1,2, \cdots, r_{e}\right\}$ and $W=\left\{1,2, \cdots, r r_{e}\right\}$, and the bijection $\phi: V \otimes H \rightarrow W$ where $\otimes$ is the Cartesian product operator. Then the T-S fuzzy descriptor system (2) can be transformed into the following system

$$
\begin{aligned}
\mathbf{E} \dot{\eta}(t) & =\left(\sum_{i=1}^{r r_{e}} w_{i}(z(t)) \mathbf{A}_{i}+\Delta \mathbf{A}\right) \eta(t)+\mathbf{B} u(t) \\
y(t) & =\mathbf{C} \eta(t)
\end{aligned}
$$

where $w_{i}(z(t)) \geq 0, i=1,2, \cdots, r r_{e}$, is the normalized fuzzy membership function with the property $\sum_{i=1}^{r r_{e}} w_{i}(z(t))=1$.

Remark 2: Without loss of generality, the bijection $\phi$ can be defined as $j=\phi(k, i)=i+(k-1) r_{e}, j=1,2, \cdots, r r_{e}, k=$ $1,2, \cdots, r_{e}, i=1,2, \cdots, r$, and the fuzzy membership function is defined as $w_{j}(z(t))=v_{k}(z(t)) h_{i}(z(t))$ with $(k, i)=\phi^{-1}(j)$. It is straight forward to check that $w_{j}(z(t)) \geq$ 0 and $\sum_{j=1}^{r r_{e}} w_{j}(z(t))=1$.

Based on the definition of multiple sum in [23], the following definitions can be formulated, see [23] for further details.

Definition 1: (Multiple sums) Multiple sums with $n_{H^{\Lambda}}$ terms in $h(z(t))$ and $n_{V^{\Lambda}}$ terms in $v(z(t))$ at time $t$ are of the following form $\Lambda_{H^{\Lambda}}=$ $\sum_{i_{1}=1}^{r} \sum_{i_{2}=1}^{r} \cdots \sum_{i_{n_{H} \Lambda}=1}^{r} h_{i_{1}}(z(t)) h_{i_{2}}(z(t)) \cdots h_{i_{H_{H}}}(z(t))$ $\Lambda_{i_{1} i_{2} \cdots i_{n_{H}}}, \Lambda_{V^{\Lambda}}=\sum_{j_{1}=1}^{H_{e}} \sum_{j_{2}=1}^{r_{e}} \cdots \sum_{j_{n_{V} \Lambda}=1}^{r_{e}} v_{j_{1}}(z(t))$ $v_{j_{2}}(z(t)) \cdots v_{j_{n \Lambda}}(z(t)) \Lambda_{j_{1} j_{2} \cdots j_{n_{V}}}$, where $H^{\Lambda}=$ $\{\underbrace{0,0, \cdots, 0}_{n_{H^{\Lambda}}}\}$ and $V^{\Lambda}=\{\underbrace{0,0, \cdots, 0}_{n_{V^{\Lambda}}}\}$ are multisets.

The cardinality of a multiset $H,|H|=n_{H}$ is defined as the number of elements in $H$. The union of two multisets $\Lambda_{A}$ and $\Lambda_{B}$ is $\Lambda_{C}=\Lambda_{A} \cup \Lambda_{B}$, such that $n_{\Lambda_{C}}=\max \left\{n_{\Lambda_{A}}, n_{\Lambda_{B}}\right\}$. The intersection of two multisets $\Lambda_{A}$ and $\Lambda_{B}$ is $\Lambda_{C}=\Lambda_{A} \cap \Lambda_{B}$, such that $n_{\Lambda_{C}}=\min \left\{n_{\Lambda_{A}}, n_{\Lambda_{B}}\right\}$. The sum of two multisets $\Lambda_{A}$ and $\Lambda_{B}$ is $\Lambda_{C}=\Lambda_{A} \oplus \Lambda_{B}$, such that $n_{\Lambda_{C}}=n_{\Lambda_{A}}+n_{\Lambda_{B}}$. The index set of a multiple sum $\Lambda_{H}$ is $\mathbb{I}_{H}=\left\{i_{1} i_{2} \cdots i_{n_{H}}: i_{1}, i_{2}, \cdots, i_{n_{H}}=1,2, \cdots, r\right\}$, the set of all indices that appear in the sum. An element $\mathbf{i}$ is a multiindex. The projection of the index $\mathbf{i} \in \mathbb{I}_{H_{A}}$ to the multiset $H_{B}, p r_{H_{B}}^{\mathrm{i}}$, is the part of the index that corresponds to the index in $H_{A} \cap H_{B}$.

Based on Definition 1, define $W^{\mathbf{A}}=\{0\}$, the descriptor system (3) is further rewritten as

$$
\begin{aligned}
\mathbf{E} \dot{\eta}(t) & =\left(\mathbf{A}_{W \mathbf{A}}+\Delta \mathbf{A}\right) \eta(t)+\mathbf{B} u(t) \\
y(t) & =\mathbf{C} \eta(t)
\end{aligned}
$$

Lemma 1: (Finsler's Lemma) [42] Let $x \in \mathbb{R}^{n}, \Omega=$ $\Omega^{T} \in \mathbb{R}^{n \times n}, W \in \mathbb{R}^{m \times n}$. The followings are equivalent: 1) $x^{T} \Omega x<0, \forall W x=0, x \neq 0$; 2) $\exists X \in \mathbb{R}^{n \times m}$ : $\Omega+X W+W^{T} X^{T}<0$.
Lemma 2: [28] Suppose a piecewise continuous matrix $A(t) \in \mathbb{R}^{n \times n}$, and a matrix $X \in \mathbb{R}^{n \times n}$ satisfy the following inequality $A(t)^{T} X+X^{T} A(t) \leq-\alpha I$ for all $t$ and some positive number $\alpha$. Then the following statements hold: 1) $A(t)$ is invertible; 2) $\left\|A^{-1}(t)\right\| \leq a$ for some $a>0$.

\section{OBSERVER-BASED SMC FOR T-S FUZZY DESCRIPTOR SYSTEMS}

In this section, an observer-based sliding mode control strategy is developed for the T-S fuzzy descriptor system with parameter uncertainties, where a two step design method and a single step design method are respectively proposed to determine the observer gains and coefficients in the switching function.

To estimate the states of the system (4), the following state observer is designed

$$
\mathbf{E} \dot{\hat{\eta}}(t)=\mathbf{A}_{W^{\mathbf{A}}} \hat{\eta}(t)+\mathbf{B} u(t)+\mathbf{L}_{W^{\mathbf{L}}}(y(t)-\mathbf{C} \hat{\eta}(t))
$$

where $\mathbf{L}_{W^{\mathbf{L}}}$ is the observer gain which will be determined later.

Define $e(t)=\eta(t)-\hat{\eta}(t)$. Then the error system can be obtained as

$$
\mathbf{E} \dot{e}(t)=\left(\mathbf{A}_{W^{\mathbf{A}}}-\mathbf{L}_{W^{\mathbf{L}}} \mathbf{C}\right) e(t)+\Delta \mathbf{A} \eta(t)
$$

Construct a sliding surface based on the state estimates from the observer (5) as follows

$$
\begin{aligned}
& \mathcal{S}=\{\hat{\eta}(t): s(t)=\mathbf{S E} \hat{\eta}(t)-\mathbf{S E} \hat{\eta}(0) \\
&\left.-\mathbf{S} \int_{0}^{t}\left(\mathbf{A}_{W^{\mathbf{A}}}+\mathbf{B} \mathbf{K}_{W^{\mathbf{K}}} \mathbf{X}_{W^{\mathbf{x}}}^{-1}\right) \hat{\eta}(\tau) d \tau=0\right\}
\end{aligned}
$$

where $\mathbf{K}_{W} \mathbf{K}, \mathbf{X}_{W^{\mathrm{x}}}$ are the coefficients in the switching function to be determined, and $\mathbf{S}$ is a parameter matrix satisfying $\operatorname{det}(\mathbf{S B}) \neq 0$.

As $e(t)=\eta(t)-\hat{\eta}(t)$, then $s(t)=s_{\eta}(t)-$ $s_{e}(t), \quad$ where $s_{\eta}(t)=\mathbf{S E} \eta(t)-\mathbf{S E} \eta(0)-$ $\mathbf{S} \int_{0}^{t}\left(\mathbf{A}_{W} \mathbf{A}+\mathbf{B K}_{W^{\mathbf{K}}} \mathbf{X}_{W}^{-1} \mathbf{x}\right) \eta(\tau) d \tau$ and $s_{e}(t)=$ $\mathbf{S E} e(t)-\mathbf{S E} e(0)-\mathbf{S} \int_{0}^{t}\left(\mathbf{A}_{W \mathbf{A}}+\mathbf{B K}_{W^{\mathbf{K}}} \mathbf{X}_{W^{\mathbf{x}}}^{-1}\right) e(\tau) d \tau$.

Therefore, the sliding surface (7) defined in the space $\hat{\eta}(t)$ can be described in the augmented space by

$$
\mathcal{S}=\left\{(\eta(t), e(t)): s_{\eta}(t)-s_{e}(t)=0\right\}
$$

When the system exhibits an ideal sliding mode, it is necessary that $s_{\eta}(t)-s_{e}(t)=0$ and $\dot{s}_{\eta}(t)-\dot{s}_{e}(t)=0$. Based on (4), (6) and (8), it can be computed that

$$
\begin{aligned}
\dot{s}(t)= & \mathbf{S B} u(t)-\mathbf{S B K}_{W^{\mathrm{K}}} \mathbf{X}_{W^{\mathrm{x}}}^{-1} \eta(t) \\
& +\mathbf{S B K}_{W^{\mathrm{K}}} \mathbf{X}_{W^{\mathbf{x}}}^{-1} e(t)+\mathbf{S L}_{W^{\mathrm{L}}} \mathbf{C} e(t)
\end{aligned}
$$

Next, the reachability problem will be solved in order to determine the sliding mode controller.

Theorem 1: Assume that matrices $\mathbf{X}_{\mathbf{i}}, \mathbf{i} \in \mathbb{I}_{W_{\mathbf{X}}}, \mathbf{K}_{\mathbf{j}}, \mathbf{j} \in$ $\mathbb{I}_{W_{\mathbf{K}}}, \mathbf{L}_{\mathbf{k}}, \mathbf{k} \in \mathbb{I}_{W_{\mathbf{L}}}$ satisfy Theorem 2 or Theorem 3 and $\varepsilon>0$. The sliding mode controller

$$
\begin{aligned}
u(t)= & \mathbf{K}_{W^{\mathbf{K}}} \mathbf{X}_{W^{\mathbf{x}}}^{-1} \hat{\eta}(t)-(\mathbf{S B})^{-1} \\
& \times\left(\left\|\mathbf{S L}_{W^{\mathbf{L}}}(y(t)-\hat{y}(t))\right\|+\varepsilon\right) \frac{s(t)}{\|s(t)\|}
\end{aligned}
$$


can drive the T-S fuzzy descriptor system (4) to the sliding surface (7) and maintain a sliding motion.

Proof: Choose the Lyapunov function candidate $V(s(t))=\frac{1}{2} s^{T}(t) s(t)$. The time derivative of $V(s(t))$ along with (9) is

$$
\begin{aligned}
\dot{V}(s(t))= & s^{T}(t)\left(\mathbf{S B} u(t)+\mathbf{S L}_{W^{\mathbf{L}}}(y(t)-\hat{y}(t))\right. \\
& \left.-\mathbf{S B K}_{W^{\mathbf{K}}} \mathbf{X}_{W^{\mathbf{x}}}^{-1} \hat{\eta}(t)\right) \leq-\varepsilon\|s(t)\|
\end{aligned}
$$

Furthermore, it follows from (11) that

$$
\frac{d\|s(t)\|}{d t}=\frac{s^{T}(t) \dot{s}(t)}{\|s(t)\|} \leq-\varepsilon
$$

which implies that $\|s(t)\|$ is decreasing function on time $t$.

Assume that it takes time $t_{s}$ to reach the sliding surface (7), integrating (12) from 0 to $t_{s}$ gives $\left\|s\left(t_{s}\right)\right\|-\|s(0)\| \leq-\varepsilon t_{s}$. Thus, the time taken to reach the sliding surface (7) satisfies $t_{s} \leq \frac{\|s(0)\|}{\varepsilon}$ Note that $s(0)=0$, then $\|s(0)\|=0$, therefore, the sliding surface (7) is attained from the beginning.

In the sliding phase, $\dot{s}_{\eta}(t)-\dot{s}_{e}(t)=0$ holds and consequently, the equivalent control can be obtained as

$$
\begin{aligned}
u_{e q}(t)= & \mathbf{K}_{W^{\mathbf{K}}} \mathbf{X}_{W^{\mathbf{x}}}^{-1} \eta(t)-\mathbf{K}_{W^{\mathbf{K}}} \mathbf{X}_{W^{\mathbf{x}}}^{-1} e(t) \\
& -(\mathbf{S B})^{-1} \mathbf{S L}_{W^{\mathbf{L}}} \mathbf{C} e(t)
\end{aligned}
$$

Substituting (13) into (4), the ideal sliding mode dynamics are

$$
\begin{aligned}
\mathbf{E} \dot{\eta}(t)= & \left(\mathbf{A}_{W^{\mathbf{A}}}+\Delta \mathbf{A}+\mathbf{B K}_{W^{\mathbf{K}}} \mathbf{X}_{W^{\mathbf{x}}}^{-1}\right) \eta(t) \\
& -\mathbf{B}\left(\mathbf{K}_{W^{\mathbf{K}}} \mathbf{X}_{W^{\mathbf{x}}}^{-1}+(\mathbf{S B})^{-1} \mathbf{S L}_{W^{\mathbf{L}}} \mathbf{C}\right) e(t) \\
\mathbf{E} \dot{e}(t)= & \left(\mathbf{A}_{W^{\mathbf{A}}}-\mathbf{L}_{W^{\mathbf{L}}} \mathbf{C}\right) e(t)+\Delta \mathbf{A} \eta(t)
\end{aligned}
$$

Due to $\hat{\eta}(t)=\eta(t)-e(t)$, then by the following nonsingular transformation

$$
\left[\begin{array}{l}
\hat{\eta}(t) \\
e(t)
\end{array}\right]=\underbrace{\left[\begin{array}{cc}
I & -I \\
0 & I
\end{array}\right]}_{T}\left[\begin{array}{l}
\eta(t) \\
e(t)
\end{array}\right],
$$

the stability of the system (14) is equivalent to that of the following system

$$
\begin{aligned}
\mathbf{E} \dot{\hat{\eta}}(t)= & \left(\mathbf{A}_{W^{\mathbf{A}}}+\mathbf{B K}_{W^{\mathbf{K}}} \mathbf{X}_{W^{\mathbf{x}}}^{-1}\right) \hat{\eta}(t) \\
& +\left(I-\mathbf{B}(\mathbf{S B})^{-1} \mathbf{S}\right) \mathbf{L}_{W^{\mathbf{L}}} \mathbf{C} e(t) \\
\mathbf{E} \dot{e}(t)= & \left(\mathbf{A}_{W^{\mathbf{A}}}+\Delta \mathbf{A}-\mathbf{L}_{W^{\mathbf{L}}} \mathbf{C}\right) e(t)+\Delta \mathbf{A} \hat{\eta}(t)
\end{aligned}
$$

Next, the stability of the ideal sliding mode dynamics will be proved and the two steps in determining the observer gains and coefficients in the switching function are provided.

Theorem 2: The system (1) has an asymptotically stable sliding motion with respect to the sliding surface (7) if the following two conditions hold:

1: there exist positive definite matrix $R_{1}$, a set of matrices $R_{4}, Y_{k i}$, and scalars $\zeta_{k i}^{E}, \zeta_{k i}^{A}, k=1,2, \cdots, r_{e}, i=1,2, \cdots, r$ such that the following LMIs hold for $k=1,2, \cdots, r_{e}, i=$ $1,2, \cdots, r$

$$
\begin{aligned}
& {\left[\mathbf{H e}\left(R_{4}^{T} A_{i}-Y_{k i} C\right)+\zeta_{k i}^{A} N_{A}^{T} N_{A}\right.} \\
& R_{1}-E_{k}^{T} R_{4}+R_{4}^{T} A_{i}-Y_{k i} C \\
& M_{A}^{T} R_{4} \\
& -M_{E}^{T} R_{4} \\
& \left.\begin{array}{ccc}
{ }^{\star} \mathbf{H e}\left(R_{4}^{T} E_{k}\right)+\zeta_{k i}^{E} N_{E}^{T} N_{E} & \star & \star \\
M_{A}^{T} R_{4} & -\zeta_{k i}^{A} I & \star \\
-M_{E}^{T} R_{4} & 0 & -\zeta_{k i}^{E} I
\end{array}\right]<0
\end{aligned}
$$

The observer gains in the observer (5) can be obtained as $\mathbf{L}_{W^{\mathbf{L}}}=\left[\begin{array}{c}0 \\ L_{V^{E}} H^{A}\end{array}\right]$ with $L_{k i}=R_{4}^{-T} Y_{k i}, k=$ $1,2, \cdots, r_{e}, i=1,2, \cdots, r, H^{A}=\{0\}, V^{E}=\{0\} ;$

2: for a given constant $\tau>0$, there exist positive definite matrices $P_{1}, Q_{1}$, a set of matrices $P_{3 k i}, P_{4 k i}, Q_{3 i}$, $Q_{4 i}, X_{1 k}, X_{2 k}, X_{3 i}, X_{4 i}, K_{1 k i}, K_{2 k i}$, and scalars $\xi_{k i}^{E}, \xi_{k i}^{A}$, $k=1,2, \cdots, r_{e}, i=1,2, \cdots, r$ such that the following LMIs hold for $k=1,2, \cdots, r_{e}, i=1,2, \cdots, r$

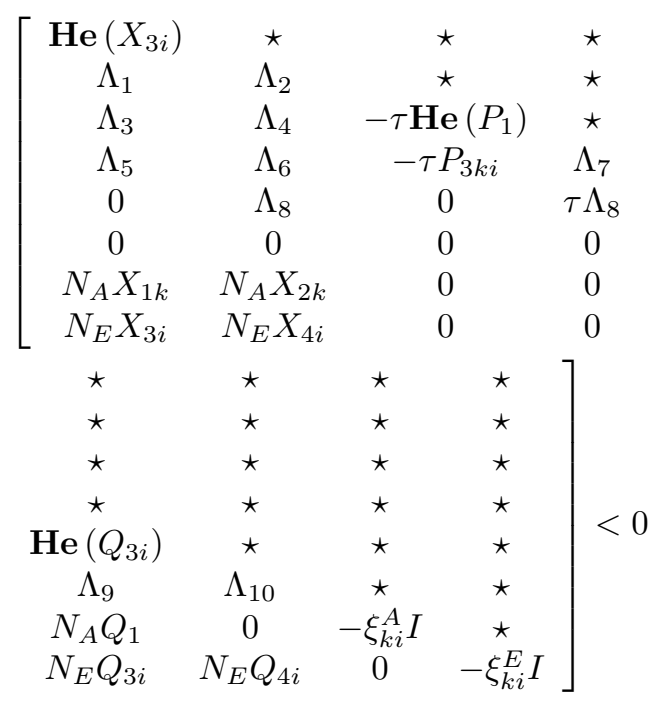

where $\Lambda_{1}=A_{i} X_{1 k}-E_{k} X_{3 i}+X_{4 i}^{T}+B K_{1 k i}, \Lambda_{2}=$ $\operatorname{He}\left(A_{i} X_{2 k}-E_{k} X_{4 i}+B K_{2 k i}\right), \quad \Lambda_{3}=X_{1 k}-P_{1}+$ $\tau X_{3 i}, \quad \Lambda_{4}=X_{2 k}+\tau X_{4 i}, \quad \Lambda_{5}=X_{3 i}-P_{3 k i}+$ $\tau\left(A_{i} X_{1 k}-E_{k} X_{3 i}+B K_{1 k i}\right), \quad \Lambda_{6}=X_{4 i}-P_{4 k i}+$ $\tau\left(A_{i} X_{2 k}-E_{k} X_{4 i}+B K_{2 k i}\right), \Lambda_{7}=-\tau \mathbf{H e}\left(P_{4 k i}\right), \Lambda_{8}=$ $Q_{1} C^{T} L_{k i}^{T} \bar{B}^{T}, \Lambda_{9}=\left(A_{i}-L_{k i} C\right) Q_{1}-E_{k} Q_{3 i}+Q_{4 i}^{T}, \Lambda_{10}=$ $-\mathbf{H e}\left(E_{k} Q_{4 i}\right)+\xi_{k i}^{E} M_{E} M_{E}^{T}+\xi_{k i}^{A} M_{A} M_{A}^{T}, \bar{B}=I-B B^{+}$, and $L_{k i}, k=1,2, \cdots, r_{e}, i=1,2, \cdots, r$, are obtained from condition 1. Then coefficients in switching function (7) are $\mathbf{K}_{W^{\mathbf{K}}}=\left[\begin{array}{ll}K_{1 V^{E} H^{A}} & K_{2 V^{E} H^{A}}\end{array}\right]$ and $\mathbf{X}_{W^{\mathbf{x}}}=$ $\left[\begin{array}{ll}X_{1 V^{E}} & X_{2 V^{E}} \\ X_{3 H^{A}} & X_{4 H^{A}}\end{array}\right]$

Proof: Suppose that there exist matrices and scalars satisfying (17) and (18) in Theorem 2, the asymptotic stability of the sliding motion (16) will be derived. Define $\mathbf{R}=$ $\left[\begin{array}{cc}R_{1} & 0_{n \times n} \\ R_{4} & R_{4}\end{array}\right], \zeta_{W_{\zeta}}=\left[\begin{array}{cc}\zeta_{V^{E} H^{A}}^{A} I & 0 \\ 0 & \zeta_{V^{E} H^{A}}^{E} I\end{array}\right], \mathbf{Y}_{W^{\mathbf{Y}}}=$ $\left[\begin{array}{c}Y_{V^{E} H^{A}} \\ Y_{V^{E} H^{A}}\end{array}\right], \mathbf{P}_{W^{\mathbf{P}}}=\left[\begin{array}{cc}P_{1} & 0_{n \times n} \\ P_{3 V^{E} H^{A}} & P_{4 V^{E} H^{A}}\end{array}\right], \mathbf{Q}_{W \mathbf{Q}}=$ $\left[\begin{array}{cc}Q_{1} & 0_{n \times n} \\ Q_{3 H^{A}} & Q_{4 H^{A}}\end{array}\right], \boldsymbol{\xi}_{W_{\boldsymbol{\xi}}}=\left[\begin{array}{cc}\xi_{V^{E} H^{A}}^{A} I & 0 \\ 0 & \xi_{V^{E} H^{A}}^{E} I\end{array}\right], \mathbf{S}=$ $\mathbf{B}^{+}$. (17) and (18) are equivalent to

$$
\left[\begin{array}{cc}
\mathbf{H e}\left(\mathbf{R}^{T} \mathbf{A}_{W^{\mathrm{A}}}-\mathbf{Y}_{W^{\mathbf{Y}}} \mathbf{C}\right)+\mathbf{N}^{T} \boldsymbol{\zeta}_{W_{\zeta}} \mathbf{N} & \mathbf{M}^{T} \mathbf{R} \\
\boldsymbol{\zeta}_{W_{\zeta}} I
\end{array}\right]<0
$$


and

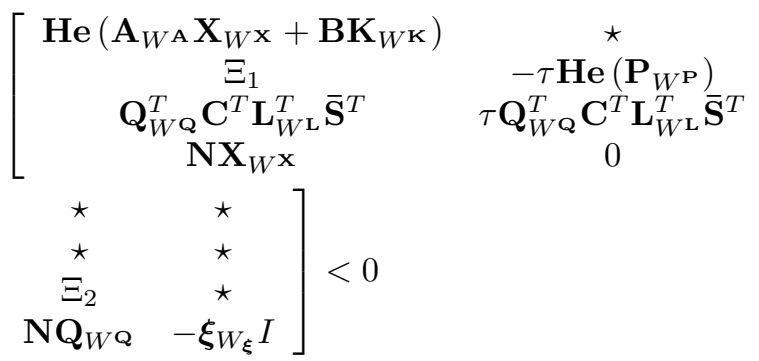

where $\Xi_{1}=\mathbf{X}_{W} \mathbf{x}-\mathbf{P}_{W \mathbf{P}}+\tau\left(\mathbf{A}_{W} \mathbf{A} \mathbf{X}_{W} \mathbf{x}+\mathbf{B K}_{W} \mathbf{K}\right)$, $\Xi_{2}=\mathbf{H e}\left(\left(\mathbf{A}_{W^{\mathrm{A}}}-\mathbf{L}_{W^{\mathrm{L}}} \mathbf{C}\right) \mathbf{Q}_{W^{\mathbf{Q}}}\right)+\mathbf{M} \boldsymbol{\xi}_{W_{\boldsymbol{\xi}}} \mathbf{M}^{T}, \overline{\mathbf{S}}=I-$ $\mathbf{B}(\mathbf{S B})^{-1} \mathbf{S}, W_{\boldsymbol{\zeta}}=W^{\mathbf{A}} \cup W^{\mathbf{Y}}$ and $W_{\boldsymbol{\xi}}=\left(W^{\mathbf{A}} \oplus W^{\mathbf{X}}\right) \cup$ $W^{\mathbf{K}} \cup W^{\mathbf{P}} \cup\left(\left(W^{\mathbf{A}} \cup W^{\mathbf{L}}\right) \oplus W^{\mathbf{Q}}\right)$.

The observer gain $\mathbf{L}_{W^{\mathbf{L}}}=\mathbf{R}^{-T} \mathbf{Y}_{W^{\mathbf{Y}}}$ can be solved by (19) which is a sufficient condition for the asymptotic stability of the following system

$$
\mathbf{E} \dot{e}(t)=\left(\mathbf{A}_{W^{\mathbf{A}}}+\Delta \mathbf{A}-\mathbf{L}_{W^{\mathbf{L}}} \mathbf{C}\right) e(t)
$$

The asymptotic stability of the system (21) can be similarly derived from that of sliding motion (16) and thus is omitted. In the sequel, it will show that if (20) holds, the sliding motion (16) is asymptotically stable.

Using the Schur complement, (20) holds if and only if the following is satisfied

$$
\Xi+\widehat{\mathbf{M}}^{T} \boldsymbol{\xi}_{W_{\boldsymbol{\xi}}} \widehat{\mathbf{M}}+\widehat{\mathbf{N}}^{T} \boldsymbol{\xi}_{W_{\boldsymbol{\xi}}}^{-1} \widehat{\mathbf{N}}<0
$$

where $\widehat{\mathbf{M}}=\left[\begin{array}{lll}0 & 0 & \mathbf{M}^{T}\end{array}\right], \quad \widehat{\mathbf{N}}=$ $\left[\begin{array}{lll}\mathbf{N X}_{W} \mathbf{x} & 0 & \mathbf{N Q}_{W}^{\mathbf{Q}}\end{array}\right]$,

$$
\begin{aligned}
& \Xi=\left[\begin{array}{cc}
\mathbf{H e}\left(\mathbf{A}_{W^{\mathbf{A}}} \mathbf{X}_{W \mathbf{x}}+\mathbf{B} \mathbf{K}_{W^{\mathbf{K}}}\right) & \star \\
\Xi_{1} & -\tau \mathbf{H e}\left(\mathbf{P}_{W^{\mathbf{P}}}\right) \\
\mathbf{Q}_{W^{\mathbf{Q}}}^{T} \mathbf{C}^{T} \mathbf{L}_{W^{\mathbf{L}}}^{T} \overline{\mathbf{S}}^{T} & \tau \mathbf{Q}_{W^{\mathbf{Q}}}^{T} \mathbf{C}^{T} \mathbf{L}_{W^{\mathbf{L}}}^{T} \overline{\mathbf{S}}^{T}
\end{array}\right. \\
& \left.\mathbf{H e}\left(\left(\mathbf{A}_{W^{\mathrm{A}}}-\mathbf{L}_{W^{\mathrm{L}}} \mathbf{C}\right) \mathbf{Q}_{W^{\mathbf{Q}}}\right)\right]
\end{aligned}
$$

Note that $\mathbf{H e}(\mathbf{M F}(t) \mathbf{N})<\mathbf{M} \boldsymbol{\xi}_{W_{\boldsymbol{\xi}}} \mathbf{M}^{T}+\mathbf{N}^{T} \boldsymbol{\xi}_{W_{\boldsymbol{\xi}}}^{-1} \mathbf{N}$, simple algebraic manipulation on (22) implies

$$
\Xi+\mathcal{X}_{1}<0
$$

where $\mathcal{X}_{1}=\mathbf{H e}\left(\left[\begin{array}{ccc}0 & 0 & 0 \\ 0 & 0 & 0 \\ \text { Pre- } & \text { and } \\ \text { post-multiplying }_{W \mathbf{x}} & 0 & \Delta \mathbf{A Q}_{W^{\mathbf{Q}}}\end{array}\right]\right)$ (23) $\operatorname{diag}\left\{\mathbf{X}_{W}^{-T} \mathbf{X}, \mathbf{P}_{W^{\mathbf{P}}}^{-1}, \mathbf{Q}_{W \mathbf{Q}}^{-T}\right\}$ and its transpose yields

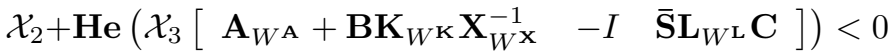
where $\mathcal{X}_{2}=\left[\begin{array}{ccc}0 & \star & \star \\ 0 & 0 & \star \\ 0 & 0 & \mathbf{H e}\left(\mathbf{Q}_{W^{\mathbf{Q}}}^{-T}\left(\mathbf{A}_{W^{\mathbf{A}}}+\Delta \mathbf{A}-\mathbf{L}_{W^{\mathbf{L}}} \mathbf{C}\right)\right)\end{array}\right]$ $+\left[\begin{array}{ccc}0 & \star & \star \\ \mathbf{P}_{W}^{-1} & 0 & \star \\ \mathbf{Q}_{W}^{-T} \Delta \mathbf{A} & 0 & 0\end{array}\right], \mathcal{X}_{3}=\left[\begin{array}{c}\mathbf{X}_{W \mathbf{X}}^{-T} \\ \tau \mathbf{P}_{W}^{-1} \\ 0\end{array}\right]$.

When (24) holds, it follows from Lemma 1 that

$$
\bar{x}^{T} \mathcal{X}_{2} \bar{x}<0
$$

for $\forall \bar{x}=\left[\begin{array}{lll}x_{1}^{T} & x_{2}^{T} & x_{3}^{T}\end{array}\right]^{T} \neq 0$ satisfying

$$
x_{2}=\left(\mathbf{A}_{W^{\mathbf{A}}}+\mathbf{B} \mathbf{K}_{W^{\mathbf{K}}} \mathbf{X}_{W^{\mathbf{x}}}^{-1}\right) x_{1}+\overline{\mathbf{S}} \mathbf{L}_{W^{\mathbf{L}}} \mathbf{C} x_{3}
$$

Substituting (26) into (25), by congruent transformation, it is obtained that for $\forall \tilde{x}=\left[\begin{array}{ll}x_{1}^{T} & x_{3}^{T}\end{array}\right]^{T} \neq 0, \tilde{x}^{T} \Upsilon \tilde{x}<0$, where $\Upsilon=\left[\begin{array}{cc}\mathbf{H e}\left(\mathbf{P}_{W^{\mathbf{P}}}^{-T}\left(\mathbf{A}_{W^{\mathbf{A}}}+\mathbf{B K}_{W^{\mathbf{K}}} \mathbf{X}_{W^{\mathbf{x}}}^{-1}\right)\right) & \star \\ \left(\overline{\mathbf{S}} \mathbf{L}_{W^{\mathbf{L}}} \mathbf{C}\right)^{T} \mathbf{P}_{W^{\mathbf{P}}}^{-1}+\mathbf{Q}_{W^{\mathbf{Q}}}^{-T} \Delta \mathbf{A} & 0\end{array}\right]+$ $\left[\begin{array}{cc}0 & \multirow{2}{*}{{ }^{\star}} \\ 0 & \mathbf{H e}\left(\mathbf{Q}_{W^{\mathbf{Q}}}^{-T}\left(\mathbf{A}_{W^{\mathbf{A}}}+\Delta \mathbf{A}-\mathbf{L}_{W^{\mathbf{L}}} \mathbf{C}\right)\right)\end{array}\right]$, which implies $\Upsilon<0$

Define

$\mathbf{A}_{W \mathbf{A}}+\mathbf{B K}_{W \mathbf{K}} \mathbf{X}_{W^{\mathbf{x}}}^{-1}=\left[\begin{array}{cc}\hat{A}_{1}^{1} & \hat{A}_{2}^{1} \\ \hat{A}_{3}^{1} & \hat{A}_{4}^{1}\end{array}\right], \hat{\eta}(t)=\left[\begin{array}{c}\hat{\eta}_{1}(t) \\ \hat{\eta}_{2}(t)\end{array}\right]$,

$\overline{\mathbf{S}} \mathbf{L}_{W^{\mathbf{L}}} \mathbf{C}=\left[\begin{array}{cc}\hat{A}_{1}^{2} & \hat{A}_{2}^{2} \\ \hat{A}_{3}^{2} & \hat{A}_{4}^{2}\end{array}\right], \Delta \mathbf{A}=\left[\begin{array}{cc}\hat{A}_{1}^{3} & \hat{A}_{2}^{3} \\ \hat{A}_{3}^{3} & \hat{A}_{4}^{3}\end{array}\right]$,

$\mathbf{A}_{W} \mathbf{A}+\Delta \mathbf{A}-\mathbf{L}_{W} \mathbf{L} \mathbf{C}=\left[\begin{array}{cc}\hat{A}_{1}^{4} & \hat{A}_{2}^{4} \\ \hat{A}_{3}^{4} & \hat{A}_{4}^{4}\end{array}\right], e(t)=\left[\begin{array}{c}e_{1}(t) \\ e_{2}(t)\end{array}\right]$

where $\hat{A}_{4}^{1} \in \mathbb{R}^{n \times n}, \hat{A}_{4}^{2} \in \mathbb{R}^{n \times n}, \hat{A}_{4}^{3} \in \mathbb{R}^{n \times n}, \hat{A}_{4}^{4} \in \mathbb{R}^{n \times n}$, $\hat{\eta}_{1}(t) \in \mathbb{R}^{n}, e_{1}(t) \in \mathbb{R}^{n}$.

Substituting (28) into (27), by Lemma 2, it can be proved that $\hat{G}_{4}$ is invertible and $\left\|\hat{G}_{4}^{-1}\right\|$ is bounded where $\hat{G}_{4}=$ $\left[\begin{array}{cc}\hat{A}_{4}^{1} & \hat{A}_{4}^{2} \\ \hat{A}_{4}^{3} & \hat{A}_{4}^{4}\end{array}\right]$. Furthermore, define $\hat{G}_{3}=\left[\begin{array}{cc}\hat{A}_{3}^{1} & \hat{A}_{3}^{2} \\ \hat{A}_{3}^{3} & \hat{A}_{3}^{4}\end{array}\right]$, from (16) and (28), it is shown that

$$
\left[\begin{array}{c}
\hat{\eta}_{2}(t) \\
e_{2}(t)
\end{array}\right]=-\hat{G}_{4}^{-1} \hat{G}_{3}\left[\begin{array}{l}
\hat{\eta}_{1}(t) \\
e_{1}(t)
\end{array}\right]
$$

Now, the asymptotic stability of the system (16) will be derived. Select the Lyapunov function candidate as follows

$$
\begin{aligned}
V\left(\hat{\eta}_{1}(t), e_{1}(t)\right)= & \hat{\eta}_{1}^{T}(t) P_{1}^{-1} \hat{\eta}_{1}(t)+e_{1}^{T}(t) Q_{1}^{-1} e_{1}(t) \\
= & \hat{\eta}^{T}(t) \mathbf{E}^{T} \mathbf{P}_{W^{\mathbf{P}}}^{-1} \hat{\eta}(t) \\
& +e^{T}(t) \mathbf{E}^{T} \mathbf{Q}_{W^{\mathbf{Q}}}^{-1} e(t)
\end{aligned}
$$

The time-derivative of the Lyapunov function along with the systems (16) can be obtained as

$$
\dot{V}\left(\hat{\eta}_{1}(t), e_{1}(t)\right)=\left[\begin{array}{ll}
\hat{\eta}^{T}(t) & e^{T}(t)
\end{array}\right] \Upsilon\left[\begin{array}{l}
\hat{\eta}(t) \\
e(t)
\end{array}\right]
$$

From (27), then $\dot{V}\left(\hat{\eta}_{1}(t), e_{1}(t)\right)<0$. Based on Lyapunov stability theory [1], $\left(\hat{\eta}_{1}(t), e_{1}(t)\right)$ is asymptotically stable. Note that $\left\|\hat{G}_{4}^{-1}\right\|$ and $\left\|\hat{G}_{3}\right\|$ are bounded, by (29), the asymptotic stability of $\left(\hat{\eta}_{2}(t), e_{2}(t)\right)$ can also be proved. Therefore, if the conditions (19) and (20) in Theorem 2 are solvable simultaneously, the system (16) is asymptotically stable, then the asymptotic stability of the ideal sliding mode dynamics (14) is proved.

Remark 3: It is noteworthy that the observer gains and the coefficients in the switching function for observer-based sliding mode control of T-S fuzzy descriptor systems (1) can be determined by the two-step approach in Theorem 2 . Since the redundancy of derivative matrix $\mathbf{E}$ is used in this paper, the block matrices $P_{3 V^{E} H^{A}}, P_{4 V^{E} H^{A}}$ in $\mathbf{P}_{W^{\mathbf{P}}}$ are dependent of membership functions $v_{k}(z(t))$ and $h_{i}(z(t))$, the 
conservativeness will be consequently reduced. Furthermore, by Finsler's Lemma, the system matrices and the Lyapunov matrices are decoupled, which facilitates the design.

Remark 4: It is noted that each local subsystem of the T-S fuzzy descriptor system shares the same input matrix and output matrix, when considering the resulting LMI conditions, the condition with one sum in $v_{k}(z(t))$ and one sum in $h_{i}(z(t))$ is used due to its simplicity. When more sums in $v_{k}(z(t))$ and $h_{i}(z(t))$ are considered, the solution space will enlarge, but more variables and LMIs will be involved. Therefore, Theorem 2 only provides the criterion by considering one sum in $v_{k}(z(t))$ and one sum in $h_{i}(z(t))$.

Consider the T-S fuzzy descriptor system in the form of (4), from the proof of Theorem 2, the following corollary can be obtained.

Corollary 1: The system (4) has an asymptotically stable sliding motion with respect to the sliding surface (7) if the following two conditions hold:

1: there exist matrices $\mathbf{R}, \mathbf{Y}_{\mathbf{j}} \mathbf{Y}, \mathbf{j}^{\mathbf{Y}} \in p r_{W^{\mathbf{Y}}}^{\mathbf{j}}, \boldsymbol{\zeta}_{\mathbf{j}}, \mathbf{j} \in \mathbb{I}_{W_{\zeta}}$ satisfying (19) with $W_{\zeta}=W^{\mathbf{A}} \cup W^{\mathbf{Y}}$ and $\mathbf{R}=\left[\begin{array}{cc}R_{1} & 0_{n \times n} \\ R_{3} & R_{4}\end{array}\right]$ with $R_{1}>0$. The observer gains can be obtained from $\mathbf{L}_{\mathbf{j}} \mathbf{Y}=\mathbf{R}^{-T} \mathbf{Y}_{\mathbf{j}} \mathbf{Y}, \mathbf{j}^{\mathbf{Y}} \in p r_{W}^{\mathbf{Y}}, \mathbf{j} \in \mathbb{I}_{W_{\zeta}}$;

2: for a given constant $\tau>0$, there exist matrices $\mathbf{P}_{\mathbf{i}^{\mathrm{P}}}$, $\mathbf{i}^{\mathbf{P}} \in p r_{W^{\mathbf{P}}}^{\mathbf{i}}, \mathbf{X}_{\mathbf{i}^{\mathbf{x}}}, \mathbf{i}^{\mathbf{X}} \in p r_{W^{\mathbf{x}}}^{\mathbf{i}}, \mathbf{K}_{\mathbf{i}^{\mathrm{K}}}, \mathbf{i}^{\mathrm{K}} \in p r_{W^{\mathrm{K}}}^{\mathrm{i}}, \mathbf{Q}_{\mathbf{i} \mathbf{Q}}$, $\mathbf{i}^{\mathbf{Q}} \in p r_{W \mathbf{Q}}^{\mathbf{i}}, \boldsymbol{\xi}_{\mathbf{i}}, \mathbf{i} \in \mathbb{I}_{W_{\xi}}$ satisfying (20) with $\mathbf{S}=I-$ $\mathbf{B}(\mathbf{S B})^{-1} \mathbf{S}, \Xi_{1}=\mathbf{X}_{W^{\mathbf{x}}}-\mathbf{P}_{W^{\mathbf{P}}}+\tau\left(\mathbf{A}_{W^{\mathbf{A}}} \mathbf{X}_{W^{\mathbf{x}}}+\mathbf{B K}_{W^{\mathbf{K}}}\right)$, $\Xi_{2}=\mathbf{H e}\left(\left(\mathbf{A}_{W^{\mathrm{A}}}-\mathbf{L}_{W^{\mathrm{L}}} \mathbf{C}\right) \mathbf{Q}_{W^{\mathbf{Q}}}\right)+\mathbf{M} \boldsymbol{\xi}_{W_{\xi}} \mathbf{M}^{T}, \mathbf{P}_{W^{\mathbf{P}}}=$ $\left[\begin{array}{cc}P_{1} & 0_{n \times n} \\ P_{3 W^{\mathbf{P}}} & P_{4 W^{\mathbf{P}}}\end{array}\right], \mathbf{Q}_{W^{\mathbf{Q}}}=\left[\begin{array}{cc}Q_{1} & 0_{n \times n} \\ Q_{3 W^{\mathbf{Q}}} & Q_{4 W^{\mathbf{Q}}}\end{array}\right]$ with $P_{1}>$ 0 and $Q_{1}>0, W_{\boldsymbol{\xi}}=\left(W^{\mathbf{A}} \oplus W^{\mathbf{X}}\right) \cup W^{\mathbf{K}} \cup W^{\mathbf{P}} \cup$ $\left(\left(W^{\mathbf{A}} \cup W^{\mathbf{L}}\right) \oplus W^{\mathbf{Q}}\right)$, and the observer gain $\mathbf{L}_{W^{\mathbf{L}}}$ defined from condition 1 .

Remark 5: Based on the definition of multiple sums in [23], Corollary 1 provides a general framework of the conditions with multiple sums for T-S fuzzy descriptor systems (4) in continuous time. By selecting the numbers of zeros in the multisets $W^{\mathbf{R}}, W^{\mathbf{K}}, W^{\mathbf{P}}, W^{\mathbf{Q}}$, conditions with different conservativeness will be obtained. The more zeros the multisets $W^{\mathbf{R}}, W^{\mathbf{K}}, W^{\mathbf{P}}, W^{\mathbf{Q}}$ contain, the less conservative the conditions will be, but at the same time, the more decision variables and LMIs will be involved. For the design purpose, only one sum in $w_{i}, i=1,2, \cdots, r r_{e}$, is considered.

Although Theorem 2 (or Corollary 1) presents an LMIbased method to determine the observer gains and the coefficients in the switching function, the two-step design approach [3] seems less convenient than the single-step approach [5]. However, when uncertainties are involved in the system matrices of the system (1) (or system (4)), the separation principle does not hold [10]. As a result, by appropriately applying Finsler's Lemma, the following single-step design approach for the observer-based stabilization problem is given.

Theorem 3: The system (1) has an asymptotically stable sliding motion with respect to the sliding surface (7) if for a given constant $\tau>0$, there exist positive definite matrices $P_{1}$, $Q_{1}$, a set of matrices $P_{3 k i}, P_{4 k i}, Q_{3 i}, Q_{4 i}, X_{1 k}, X_{2 k}, X_{3 i}$, $X_{4 i}, K_{1 k i}, K_{2 k i}, Y_{k i}, R_{1}^{1}, R_{1 k i}^{3}, R_{1 k i}^{4}, R_{2 k i}^{1}, R_{3 k i}, R_{4 k i}$, and scalars $\xi_{k i}^{E}, \xi_{k i}^{A}, k=1,2, \cdots, r_{e}, i=1,2, \cdots, r$, such that the following LMIs hold for $k=1,2, \cdots, r_{e}, i=1,2, \cdots, r$,

\begin{tabular}{|c|c|c|c|c|c|}
\hline $\mathbf{H e}\left(X_{3 i}\right)$ & & $\star$ & $\star$ & $\star$ & $\star$ \\
\hline$\Pi_{1}$ & & $\Pi_{2}$ & $\star$ & $\star$ & $\star$ \\
\hline$\Pi_{3}$ & & $\Pi_{4}$ & $\Pi_{5}$ & $\star$ & $\star$ \\
\hline$\Pi_{6}$ & & $\Pi_{7}$ & $-\tau P_{3 k i}$ & $\Pi_{8}$ & $\star$ \\
\hline 0 & & $\Pi_{9}$ & 0 & $\tau \Pi_{9}$ & $\mathbf{H e}\left(Q_{3 i}\right)$ \\
\hline 0 & & 0 & 0 & 0 & $\Pi_{10}$ \\
\hline 0 & & $v \Pi_{9}$ & 0 & $\tau v \Pi_{9}$ & $\Pi_{12}$ \\
\hline 0 & & 0 & 0 & 0 & $\Pi_{15}$ \\
\hline$N_{A} X_{1 k}$ & & $v_{A} X_{2 k}$ & 0 & 0 & $N_{A} Q_{1}$ \\
\hline$N_{E} X_{3 i}$ & & $V_{E} X_{4 i}$ & 0 & 0 & $N_{E} Q_{3 i}$ \\
\hline$\star$ & $\star$ & $\star$ & $\star$ & $\star$ & \\
\hline$\star$ & $\star$ & $\star$ & $\star$ & $\star$ & \\
\hline$\star$ & $\star$ & $\star$ & $\star$ & $\star$ & \\
\hline$\star$ & $\star$ & $\star$ & $\star$ & $\star$ & \\
\hline$\star$ & $\star$ & $\star$ & $\star$ & $\star$ & $<0$ \\
\hline$\Pi_{11}$ & $\star$ & $\star$ & $\star$ & $\star$ & $<0$ \\
\hline$\Pi_{13}$ & $\Pi_{14}$ & $\star$ & $\star$ & $\star$ & \\
\hline$\Pi_{16}$ & $\Pi_{17}$ & $\Pi_{18}$ & $\star$ & $\star$ & \\
\hline 0 & 0 & 0 & $-\xi_{k i}^{A} I$ & $\star$ & \\
\hline$N_{E} Q_{4 i}$ & 0 & 0 & 0 & $\xi_{k i}^{E} I$ & \\
\hline
\end{tabular}

where $\Pi_{1}=A_{i} X_{1 k}-E_{k} X_{3 i}+X_{4 i}^{T}+B K_{1 k i}, \Pi_{2}=$ $\operatorname{He}\left(A_{i} X_{2 k}-E_{k} X_{4 i}+B K_{2 k i}\right), \Pi_{3}=X_{1 k}-P_{1}+\tau X_{3 i}$, $\Pi_{4}=X_{2 k}+\tau X_{4 i}, \Pi_{5}=-\tau \mathbf{H e}\left(P_{1}\right), \Pi_{6}=X_{3 i}-$ $P_{3 k i}+\tau\left(A_{i} X_{1 k}-E_{k} X_{3 i}+B K_{1 k i}\right), \Pi_{7}=X_{4 i}-P_{4 k i}+$ $\tau\left(A_{i} X_{2 k}-E_{k} X_{4 i}+B K_{2 k i}\right), \Pi_{8}=-\tau \mathbf{H e}\left(P_{4 k i}\right), \Pi_{9}=$ $\bar{Y}_{k i}^{T} \bar{B}^{T}, \Pi_{10}=A_{i} Q_{1}-\bar{Y}_{k i}-E_{k} Q_{3 i}+Q_{4 i}^{T}, \Pi_{11}=$ $-\mathbf{H e}\left(E_{k} Q_{4 i}\right)+\xi_{k i}^{E} M_{E} M_{E}^{T}+\xi_{k i}^{A} M_{A} M_{A}^{T}, \Pi_{12}=Q_{1}-U R_{1 k i}$, $\Pi_{13}=-v \bar{Y}_{k i}^{T}-U R_{2 k i}, \Pi_{14}=-v \mathbf{H e}\left(U R_{1 k i}\right), \Pi_{15}=Q_{3 i}-$ $R_{3 k i}, \Pi_{16}=Q_{4 i}-R_{4 k i}, \Pi_{17}=-v\left(R_{3 k i}+R_{2 k i}^{T} U^{T}\right), \Pi_{18}=$ $-v \mathbf{H e}\left(R_{4 k i}\right), \bar{B}=I-B B^{+}, U=\left[\begin{array}{ll}C^{T}\left(C C^{T}\right)^{-1} & C^{\perp}\end{array}\right]$, $\bar{Y}_{k i}=\left[\begin{array}{ll}Y_{k i} & 0_{n \times(n-l)}\end{array}\right], R_{1 k i}=\left[\begin{array}{cc}R_{1}^{1} & 0_{l \times(n-l)} \\ R_{1 k i}^{3} & R_{1 k i}^{4}\end{array}\right]$, $R_{2 k i}=\left[\begin{array}{c}0_{l \times n} \\ R_{2 k i}^{1}\end{array}\right]$. Moreover, the observer gain in the observer (5) and coefficient in the switching function (7) can be obtained as $\mathbf{L}_{W^{\mathbf{L}}}=\left[\begin{array}{c}0 \\ L_{V^{E} H^{A}}\end{array}\right], \mathbf{K}_{W^{\mathbf{K}}}=$ $\left[\begin{array}{ll}K_{1 V^{E} H^{A}} & K_{2 V^{E} H^{A}}\end{array}\right]$ and $\mathbf{X}_{W^{\mathbf{x}}}=\left[\begin{array}{ll}X_{1 V^{E}} & X_{2 V^{E}} \\ X_{3 H^{A}} & X_{4 H^{A}}\end{array}\right]$ with $L_{k i}=Y_{k i}\left(R_{1}^{1}\right)^{-1}, k=1,2, \cdots, r_{e}, i=1,2, \cdots, r$, $H^{A}=\{0\}, V^{E}=\{0\}$.

Proof: From the proof of Theorem 2, (23) is a sufficient condition for the asymptotic stability of the ideal sliding mode dynamics (14). Therefore, if (23) can be guaranteed by the LMIs in (32), then the asymptotic stability of the ideal sliding mode dynamics (14) will be proved.

$$
\begin{aligned}
& \text { Define } \quad \mathbf{P}_{W^{\mathbf{P}}}=\left[\begin{array}{cc}
P_{1} & 0_{n \times n} \\
P_{3 V^{E} H^{A}} & P_{4 V^{E} H^{A}}
\end{array}\right], \quad \mathbf{Q}_{W^{\mathbf{Q}}}= \\
& {\left[\begin{array}{cc}
Q_{1} & 0_{n \times n} \\
Q_{3 H^{A}} & Q_{4 H^{A}}
\end{array}\right], \quad \mathbf{R}_{W^{\mathrm{R}}}=\left[\begin{array}{cc}
R_{1 V^{E} H^{A}} & R_{2 V^{E} H^{A}} \\
R_{3 V^{E} H^{A}} & R_{4 V^{E} H^{A}}
\end{array}\right],} \\
& \boldsymbol{\xi}_{W_{\boldsymbol{\xi}}}=\left[\begin{array}{cc}
\xi_{V^{E} H^{A}}^{A} I & 0 \\
0 & \xi_{V^{E} H^{A}}^{E} I
\end{array}\right], \quad \mathbf{Y}_{W^{\mathbf{Y}}}= \\
& {\left[\left(\begin{array}{c}
0_{n \times l} \\
Y_{V^{E} H^{A}}
\end{array}\right) 0_{2 n \times(2 n-l)}\right], \mathbf{U}=\left[\begin{array}{cc}
U & 0 \\
0 & I_{n}
\end{array}\right], \mathbf{S}=\mathbf{B}^{+} \text {, }}
\end{aligned}
$$


simple matrix manipulation implies that (32) is equivalent to

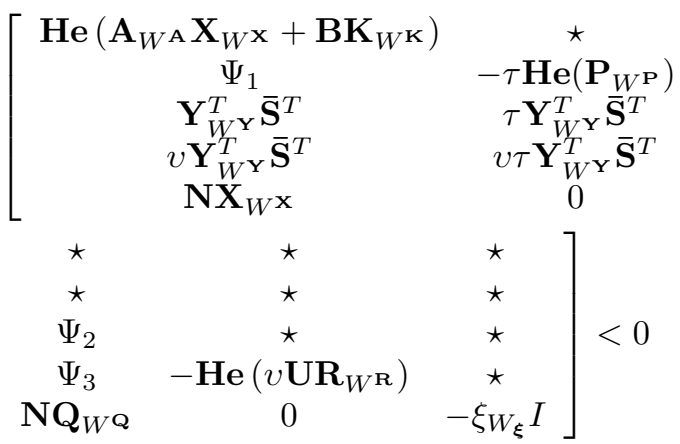

where $\Psi_{1}=\mathbf{X}_{W} \mathbf{x}-\mathbf{P}_{W} \mathbf{P}+\tau\left(\mathbf{A}_{W} \mathbf{A} \mathbf{X}_{W} \mathbf{x}+\mathbf{B K}_{W} \mathbf{K}\right)$, $\Psi_{2}=\mathbf{H e}\left(\mathbf{A}_{W} \mathbf{A} \mathbf{Q}_{W} \mathbf{Q}-\mathbf{Y}_{W} \mathbf{Y}\right)+\mathbf{M} \xi_{W_{\boldsymbol{\xi}}} \mathbf{M}^{T}, \Psi_{3}=\mathbf{Q}_{W} \mathbf{Q}-$ $v \mathbf{Y}_{W}^{T} \mathbf{Y}-\mathbf{U R}_{W} \mathbf{R}, \overline{\mathbf{S}}=I-\mathbf{B}(\mathbf{S B})^{-1} \mathbf{S}, W_{\boldsymbol{\xi}}=\left(W^{\mathbf{A}} \oplus W^{\mathbf{X}}\right) \cup$ $W^{\mathbf{K}} \cup W^{\mathbf{P}} \cup\left(W^{\mathbf{A}} \oplus W^{\mathbf{Q}}\right) \cup W^{\mathbf{Y}} \cup W^{\mathbf{R}}$. U satisfies $\mathbf{C U}=$ $\left[\begin{array}{ll}I_{l} & 0\end{array}\right]$.

By the Schur complement, (33) is equivalent to

$$
\Psi+\widetilde{\mathbf{M}}^{T} \boldsymbol{\xi}_{W_{\boldsymbol{\xi}}} \widetilde{\mathbf{M}}+\widetilde{\mathbf{N}}^{T} \boldsymbol{\xi}_{W_{\boldsymbol{\xi}}}^{-1} \widetilde{\mathbf{N}}<0
$$

where $\widetilde{\mathbf{M}}=\left[\begin{array}{llll}0 & 0 & \mathbf{M}^{T} & 0\end{array}\right]^{T}, \quad \tilde{\mathbf{N}}=$ $\left[\begin{array}{llll}\mathbf{N X}_{W} \mathbf{x} & 0 & \mathbf{N Q}_{W} \mathbf{Q} & 0\end{array}\right]$,

$$
\begin{gathered}
\Psi=\left[\begin{array}{cc}
\mathbf{H e}\left(\mathbf{A}_{W^{\mathrm{A}}} \mathbf{X}_{W^{\mathbf{x}}}+\mathbf{B K}_{W^{\mathbf{K}}}\right) & \star \\
\Psi_{1} & -\tau \mathbf{H e}\left(\mathbf{P}_{W^{\mathbf{P}}}\right) \\
\mathbf{Y}_{W}^{T} \overline{\mathbf{S}}^{T} & \tau \mathbf{Y}_{W}^{T} \overline{\mathbf{S}}^{T} \\
v \mathbf{Y}_{W}^{T} \overline{\mathbf{S}}^{T} & v \tau \mathbf{Y}_{W}^{T} \overline{\mathbf{S}}^{T} \\
\star & \star \\
\star & \star \\
\mathbf{H e}\left(\mathbf{A}_{W^{\mathrm{A}}} \mathbf{Q}_{W \mathbf{Q}}^{\mathbf{Q}}-\mathbf{Y}_{W} \mathbf{Y}\right) & \star \\
\Psi_{3} & -\mathbf{H e}\left(v \mathbf{U} \mathbf{R}_{W^{\mathbf{R}}}\right)
\end{array}\right] .
\end{gathered}
$$

Note that $\Delta \mathbf{A}=\mathbf{M F}(t) \mathbf{N}$, then (34) implies

$$
\Psi+\mathcal{Y}_{1}<0
$$

where $\mathcal{Y}_{1}=\mathbf{H e}\left(\left[\begin{array}{cccc}0 & 0 & 0 & 0 \\ 0 & 0 & 0 & 0 \\ \Delta \mathbf{A} \mathbf{X}_{W^{\mathbf{x}}} & 0 & \Delta \mathbf{A} \mathbf{Q}_{W^{\mathbf{Q}}} & 0 \\ 0 & 0 & 0 & 0\end{array}\right]\right)$.

Since $\mathbf{C U}=\left[\begin{array}{ll}I_{l} & 0\end{array}\right]$ and $\mathbf{Y}_{W^{\mathbf{Y}}}=\left[\begin{array}{ll}\mathbf{L}_{W^{\mathbf{L}}} R_{1} & 0\end{array}\right]=$ $\mathbf{L}_{W^{\mathbf{L}}}\left[\begin{array}{ll}I_{l} & 0\end{array}\right] \mathbf{R}_{W^{\mathbf{R}}}$, (35) can be rearranged as

$$
\begin{aligned}
& \mathbf{H e}\left(\mathcal{Y}_{3}\left[\mathbf{C}^{T} \mathbf{L}_{W^{\mathbf{L}}}^{T} \overline{\mathbf{S}}^{T} \tau \mathbf{C}^{T} \mathbf{L}_{W^{\mathbf{L}}}^{T} \overline{\mathbf{S}}^{T}-\mathbf{C}^{T} \mathbf{L}_{W^{\mathbf{L}}}^{T}-I\right]\right) \\
& +\mathcal{Y}_{1}+\mathcal{Y}_{2}<0
\end{aligned}
$$

where $\mathcal{Y}_{3}=\left[\begin{array}{lllll}0 & 0 & \mathbf{U R}_{W^{\mathbf{R}}} & v \mathbf{U R}_{W^{\mathbf{R}}}\end{array}\right]^{T}$ and

$$
\begin{gathered}
\mathcal{Y}_{2}=\left[\begin{array}{cc}
\mathbf{H e}\left(\mathbf{A}_{W} \mathbf{A} \mathbf{X}_{W} \mathbf{x}+\mathbf{B} \mathbf{K}_{W} \mathbf{K}\right) & \star \\
\left(\begin{array}{c}
\tau\left(\mathbf{A}_{W} \mathbf{A} \mathbf{X}_{W} \mathbf{x}+\mathbf{B} \mathbf{K}_{W} \mathbf{K}\right. \\
+\mathbf{X}_{W} \mathbf{x}-\mathbf{P}_{W} \mathbf{P}
\end{array}\right) & -\tau \mathbf{H e}\left(\mathbf{P}_{W^{\mathbf{P}}}\right) \\
0 & 0 \\
0 & 0 \\
\star & \star \\
\star & \star \\
\mathbf{H e}\left(\mathbf{A}_{W^{\mathbf{A}}} \mathbf{Q}_{W} \mathbf{Q}\right) & \star \\
\mathbf{Q}_{W} \mathbf{Q} & 0
\end{array}\right] .
\end{gathered}
$$

If (36) holds, then the following can be obtained by Lemma 1

$$
\mathbf{x}^{T}\left(\mathcal{Y}_{1}+\mathcal{Y}_{2}\right) \mathbf{x}<0
$$

for $\forall \mathbf{x}=\left[\begin{array}{llll}x_{1}^{T} & x_{2}^{T} & x_{3}^{T} & x_{4}^{T}\end{array}\right]^{T} \neq 0$ satisfying

$$
x_{4}=\mathbf{C}^{T} \mathbf{L}_{W^{\mathbf{L}}}^{T} \overline{\mathbf{S}}^{T} x_{1}+\tau \mathbf{C}^{T} \mathbf{L}_{W^{\mathbf{L}}}^{T} \overline{\mathbf{S}}^{T} x_{2}-\mathbf{C}^{T} \mathbf{L}_{W^{\mathbf{L}}}^{T} x_{3}
$$

Substituting (38) into (37), the following result can be obtained for $\forall \overline{\mathbf{x}}=\left[\begin{array}{lll}x_{1}^{T} & x_{2}^{T} & x_{3}^{T}\end{array}\right]^{T} \neq 0$

$$
\overline{\mathbf{x}}^{T}\left(\Xi+\mathcal{X}_{1}\right) \overline{\mathbf{x}}<0
$$

which implies that (23) holds. Then the asymptotic stability of the ideal sliding mode dynamics (14) can be directly derived from the proof of Theorem 2. Therefore, it suffices to know that if the LMIs in (32) hold, the ideal sliding mode dynamics (14) are asymptotically stable.

Based on the proof of Theorem 3, the following corollary can be obtained for the T-S fuzzy descriptor system in the form of (4).

Corollary 2: The system (4) has an asymptotically stable sliding motion with respect to the sliding surface (7) if for given constants $\tau>0, v>0$ there exist matrices $\mathbf{P}_{\mathbf{i}^{\mathbf{P}}}, \mathbf{i}^{\mathbf{P}} \in p r_{W^{\mathbf{P}}}^{\mathbf{i}}, \mathbf{X}_{\mathbf{i}} \mathbf{x}, \mathbf{i}^{\mathbf{X}} \in p r_{W}^{\mathbf{i}}, \mathbf{K}_{\mathbf{i}^{\mathbf{K}}}, \mathbf{i}^{\mathbf{K}} \in p r_{W^{\mathbf{K}}}^{\mathbf{i}}$, $\mathbf{Q}_{\mathbf{i} \mathbf{Q}}, \mathbf{i}^{\mathbf{Q}} \in p r_{W}^{\mathbf{i}}, \mathbf{R}_{\mathbf{i}^{\mathbf{R}}}, \mathbf{i}^{\mathbf{R}} \in p r_{W}^{\mathbf{i}}, \mathbf{Y}_{\mathbf{i}^{\mathbf{Y}}}, \mathbf{i}^{\mathbf{Y}} \in p r_{W}^{\mathbf{i}}$, $\boldsymbol{\xi}_{\mathbf{i}}$, $\mathbf{i} \in \mathbb{I}_{W_{\boldsymbol{\xi}}}$ satisfying (33) where $\overline{\mathbf{S}}=I-\mathbf{B}(\mathbf{S B})^{-1} \mathbf{S}$, $\Psi_{1}=\mathbf{X}_{W} \mathbf{x}-\mathbf{P}_{W^{\mathbf{P}}}+\tau\left(\mathbf{A}_{W} \mathbf{A} \mathbf{X}_{W} \mathbf{x}+\mathbf{B} \mathbf{K}_{W} \mathbf{K}\right), \Psi_{2}=$ $\mathbf{H e}\left(\mathbf{A}_{W} \mathbf{A} \mathbf{Q}_{W} \mathbf{Q}-\mathbf{Y}_{W} \mathbf{Y}\right)+\mathbf{M} \xi_{W_{\boldsymbol{\xi}}} \mathbf{M}^{T}, \Psi_{3}=\mathbf{Q}_{W} \mathbf{Q}-$ $v \mathbf{Y}_{W}^{T} \mathbf{Y}-\mathbf{U R}_{W^{\mathbf{R}}}, \mathbf{Y}_{W^{\mathbf{Y}}}=\left[\begin{array}{cc}Y_{1 W} \mathbf{Y} & 0_{2 n \times(2 n-l)}\end{array}\right], \mathbf{P}_{W^{\mathbf{P}}}=$ $\left[\begin{array}{cc}P_{1} & 0_{n \times n} \\ P_{3 W^{\mathbf{P}}} & P_{4 W^{\mathbf{P}}}\end{array}\right], \mathbf{Q}_{W^{\mathbf{Q}}}=\left[\begin{array}{cc}Q_{1} & 0_{n \times n} \\ Q_{3 W^{\mathbf{Q}}} & Q_{4 W^{\mathbf{Q}}}\end{array}\right], \mathbf{R}_{W^{\mathbf{R}}}=$ $\left[\begin{array}{cc}R_{1} & 0_{l \times(2 n-l)} \\ R_{3 W^{\mathbf{R}}} & R_{4 W^{\mathbf{R}}}\end{array}\right]$ with $P_{1}>0$ and $Q_{1}>0, W_{\boldsymbol{\xi}}=$ $\left(W^{\mathbf{A}} \oplus W^{\mathbf{X}}\right) \cup W^{\mathbf{K}} \cup W^{\mathbf{P}} \cup\left(W^{\mathbf{A}} \oplus W^{\mathbf{Q}}\right) \cup W^{\mathbf{Y}} \cup W^{\mathbf{R}} . \mathbf{U}$ is a nonsingular matrix such that $\mathbf{C U}=\left[\begin{array}{ll}I_{l} & 0\end{array}\right]$. The observer gains can be obtained by $\mathbf{L}_{\mathbf{i} \mathbf{Y}}=Y_{1 \mathbf{i}} \mathbf{Y} R_{1}^{-1}, \mathbf{i}^{\mathbf{Y}} \in p r_{W}^{\mathbf{i}}$, $\mathbf{i} \in \mathbb{I}_{W_{\xi}}$.

Remark 6: Theorem 3 (or Corollary 2) gives a singlestep design approach to simultaneously determine the observer gains and coefficients in the switching function for the observer-based stabilization of the T-S fuzzy descriptor system (1) (or the T-S fuzzy descriptor system (4)). Compared with Theorem 2 (or Corollary 1), the single-step design approach in Theorem 3 (or Corollary 2) is more straightforward to implement. It is also worth mentioning that since the matrix $\mathbf{R}_{U^{\mathbf{R}}}$ is selected as a lower triangular matrix, the single-step design approach may be more conservative than the two-step design approach. As a result, if the LMIs in Theorem 3 (or Corollary 2) are infeasible, the two-step approach in Theorem 2 (or Corollary 1) can be applied.

When the states of the system (4) are accessible, construct the following sliding surface

$$
\begin{aligned}
\mathcal{S}=\{ & \eta(t): s(t)=\mathbf{S E} \eta(t)-\mathbf{S E} \eta(0) \\
& \left.-\mathbf{S} \int_{0}^{t}\left(\mathbf{A}_{W} \mathbf{A}+\mathbf{B K}_{W^{\mathbf{K}}} \mathbf{X}_{W^{\mathbf{x}}}^{-1}\right) \eta(\tau) d \tau=0\right\}
\end{aligned}
$$

where $\mathbf{K}_{W} \mathbf{K}, \mathbf{X}_{W} \mathbf{x}$ and $\mathbf{S}$ share the same meanings with that in (7).

The sliding mode controller can be designed using the following result. 
Corollary 3: Assume that matrices $\mathbf{X}_{\mathbf{i}}, \mathbf{i} \in \mathbb{I}_{W_{\mathbf{X}}}, \mathbf{K}_{\mathbf{j}}$, $\mathbf{j} \in \mathbb{I}_{W_{\mathbf{K}}}$ satisfy Corollary 4 and $\varepsilon>0$. The sliding mode controller

$u(t)=\mathbf{K}_{W^{\mathbf{K}}} \mathbf{X}_{W^{\mathbf{x}}}^{-1} \eta(t)-(\|\mathbf{S M}\|\|\mathbf{N} \eta(t)\|+\varepsilon)(\mathbf{S B})^{-1} \frac{s(t)}{\|s(t)\|}$

can drive the T-S fuzzy descriptor system (4) to the sliding surface (39) and maintain a sliding motion.

The sliding mode dynamics are obtained as

$$
\begin{aligned}
\mathbf{E} \dot{\eta}(t)= & \left(\mathbf{A}_{W^{\mathbf{A}}}+\mathbf{B K}_{W^{\mathrm{K}}} \mathbf{X}_{W^{\mathbf{x}}}^{-1}\right. \\
& \left.+\left(I-\mathbf{B}(\mathbf{S B})^{-1} \mathbf{S}\right) \Delta \mathbf{A}\right) \eta(t)
\end{aligned}
$$

In this case, the unknown coefficients in the switching function can be solved by the followings.

Corollary 4: The system (1) has an asymptotically stable sliding motion with respect to the sliding surface (39) if for a given constant $\tau>0$, there exist positive definite matrix $P_{1}$, a set of matrices $P_{3 k i}, P_{4 k i}, X_{1 k}, X_{2 k}, X_{3 i}, X_{4 i}, K_{1 k i}$, $K_{2 k i}$, and scalars $\xi_{k i}^{E}, \xi_{k i}^{A}, k=1,2, \cdots, r_{e}, i=1,2, \cdots, r$, such that the following LMIs hold for $k=1,2, \cdots, r_{e}, i=$ $1,2, \cdots, r$

$$
\left.\begin{array}{ccc}
\mathbf{H e}\left(X_{3 i}\right) & \star & \star \\
\Phi_{1} & \Phi_{2} & \star \\
\Phi_{3} & \Phi_{4} & -\tau \mathbf{H e}\left(P_{1}\right) \\
\Phi_{5} & \Phi_{6} & -\tau P_{3 k i} \\
N_{A} X_{1 k} & N_{A} X_{2 k} & 0 \\
N_{E} X_{3 i} & N_{E} X_{4 i} & 0 \\
\star & \star & \star \\
\star & \star & \star \\
\star & \star & \star \\
\Phi_{7} & \star & \star \\
0 & -\xi_{k i}^{A} I & \star \\
0 & 0 & -\xi_{k i}^{E} I
\end{array}\right]<0
$$

where $\Phi_{1}=A_{i} X_{1 k}-E_{k} X_{3 i}+X_{4 i}^{T}+B K_{1 k i}$, $\Phi_{3}=X_{1 k}-P_{1}+\tau X_{3 i}, \quad \Phi_{4}=X_{2 k}+\tau X_{4 i}$, $\Phi_{2} \quad=\quad \operatorname{He}\left(A_{i} X_{2 k}-E_{k} X_{4 i}+B K_{2 k i}\right) \quad+$ $\bar{B}\left(\xi_{k i}^{E} M_{E} M_{E}^{T}+\xi_{k i}^{A} M_{A} M_{A}^{T}\right) \bar{B}^{T}, \quad \Phi_{5}=X_{3 i}-P_{3 k i}+$ $\tau\left(A_{i} X_{1 k}-E_{k} X_{3 i}+B K_{1 k i}\right), \quad \Phi_{7}=-\tau \mathbf{H e}\left(P_{4 k i}\right)+$ $\tau^{2} \bar{B}\left(\xi_{k i}^{E} M_{E} M_{E}^{T}+\xi_{k i}^{A} M_{A} M_{A}^{T}\right) \bar{B}^{T}, \quad \bar{B}=I-B B^{+}$, $\Phi_{6}=X_{4 i}-P_{4 k i}+\tau\left(A_{i} X_{2 k}-E_{k} X_{4 i}+B K_{2 k i}\right)+$ $\tau \bar{B}\left(\xi_{k i}^{E} M_{E} M_{E}^{T}+\xi_{k i}^{A} M_{A} M_{A}^{T}\right) \bar{B}^{T}$. Then coefficients in switching function (39) are $\mathbf{K}_{W \mathrm{~K}}=$ $\left[\begin{array}{ll}K_{1 V^{E} H^{A}} & K_{2 V^{E} H^{A}}\end{array}\right]$ and $\mathbf{X}_{W^{\mathbf{x}}}=\left[\begin{array}{cc}X_{1 V^{E}} & X_{2 V^{E}} \\ X_{3 H^{A}} & X_{4 H^{A}}\end{array}\right]$.

Proof: The proof can be directly derived from that of Theorem 2 and thus is omitted.

Remark 7: It is noted that when the T-S fuzzy descriptor system (1) is considered, Corollaries 3 and 4 provide a proportional and derivative feedback sliding mode control strategy to stabilize the T-S fuzzy descriptor system (1). When the derivatives of the states are unavailable, by setting $K_{2 k i}=0$, $X_{2 k}=0, k=1,2, \cdots, r_{e}, i=1,2, \cdots, r$, a proportional feedback sliding mode control strategy can be obtained.

Remark 8: As pointed out in [20], a logarithmically spaced search $\tau, v \in\left\{10^{-6}, 10^{-5}, \cdots, 10^{6}\right\}$ is used in this paper to avoid optimization technique to search for $\tau$ and $v$.

\section{Application to A Class of Mechanical Systems}

Consider a class of mechanical systems in the following nonlinear descriptor system representation

$\begin{aligned}\left(E(x(t))+\Delta E_{1}\right) \dot{x}(t) & =\left(A(x(t))+\Delta A_{1}\right) x(t)+B u(t) \\ y(t) & =C x(t)\end{aligned}$

where $x(t) \in \mathbb{R}^{n}$ is the state vector, $u(t) \in \mathbb{R}^{m}$ is the control input vector, $y(t) \in \mathbb{R}^{l}$ is the measurable output vector. $E(x(t)), A(x(t))$ are smooth nonlinear matrix functions defined in a compact set $\Omega$ of the state space containing the origin and $E(x(t))+\Delta E_{1}$ is nonsingular for $\forall x(t) \in \Omega$. $B$ and $C$ are known constant matrices of full rank. $\Delta E_{1}=$ $M_{E_{1}} F_{E_{1}}(t) N_{E_{1}}$ and $\Delta A_{1}=M_{A_{1}} F_{A_{1}}(t) N_{A_{1}}$ are parameter uncertainties where $F_{E_{1}}^{T}(t) F_{E_{1}}(t) \leq 0, F_{A_{1}}^{T}(t) F_{A_{1}}(t) \leq 0$.

In practice, some mechanical systems, such as, double inverted pendulum model of human standing in [43], twowheeled self-balanced transporter in [44] and 2-DOF planar parallel robot in [45], can be modelled as nonlinear descriptor systems (43). Applying the established sliding mode control methodology [7], [9], [12], [35], [36] to system (43) involves transformatiom to the nonlinear normal system described by

$$
\begin{aligned}
\dot{x}(t)= & \left(E(x(t))+\Delta E_{1}\right)^{-1}\left(A(x(t))+\Delta A_{1}\right) x(t) \\
& +\left(E(x(t))+\Delta E_{1}\right)^{-1} B u(t)
\end{aligned}
$$

Then the following T-S fuzzy representation of the nonlinear normal system (44) can be obtained

$$
\dot{x}(t)=\sum_{i=1}^{s} \varpi_{i}(z(t))\left(\left(\tilde{A}_{i}+\Delta \tilde{A}\right) x(t)+\left(\tilde{B}_{i}+\Delta \tilde{B}\right) u(t)\right)
$$

Since the input matrices arising in the system (45) are different, the traditional sliding mode control strategy [4] for a T-S fuzzy normal system can not be applied. Consequently, [7], [8], [11] respectively presented effective ways to solve this problem assuming that full state information is available. For mechanical systems, this assumption may be restrictive because it may be possible to only measure a sub-set of the states in practice. It should be noted that the difference in the input matrices in (45) results from the inverse operation of the derivative-term coefficient $E(x(t))$, which makes the sliding mode control problem difficult. In addition, the descriptor model of the system (43) has a more simple structure than its normal model (44) and inverse operation of derivative-term matrix is avoid.

Based on the methods proposed in Section III, a sliding mode control synthesis for the class of mechanical systems (43) can be developed without the requirement of the above inverse operation and the availability of full state information.

Assume that there exist $p_{e}$ nonlinear functions on the lefthand side and $p$ nonlinear functions on the right-hand side. Using the sector nonlinear approach, the $p_{e}$ nonlinear functions in the left-hand side can be exactly captured in the compact set $\Omega$ by the membership functions $v_{k}(z(t)), k=1,2, \cdots, 2^{p_{e}}$, and the $p$ nonlinear functions in the right-hand side by the membership functions $h_{i}(z(t)), i=1,2, \cdots, 2^{p}$. Define $r_{e}=2^{p_{e}}, r=2^{p}$, the membership functions $v_{k}(z(t)) \geq 0$, $k=1,2, \cdots, r_{e}$, and $h_{i}(z(t)) \geq 0, i=1,2, \cdots, r$, satisfy $\sum_{k=1}^{r_{e}} v_{k}(z(t))=1$ and $\sum_{i=1}^{r} h_{i}(z(t))=1$, respectively. 
Then the nonlinear descriptor system (43) can be exactly represented in the compact set $\Omega$ by the T-S fuzzy descriptor system (1).

Remark 9: As pointed out in [14], using the T-S descriptor representation, the number of fuzzy rules will decrease so that the number of LMI conditions for controller design is significantly reduced. In this paper, the advantage of the adoption of the T-S descriptor representation is that inherent characteristics of the original system are kept and yet different input matrices for each local subsystem of the T-S fuzzy system are not introduced.

Remark 10: Since the sector nonlinearity approach is used to exactly represent the nonlinear descriptor system (43) in a compact set by a T-S fuzzy descriptor system (1), the obtained T-S fuzzy descriptor system (1) is only a local representation of the nonlinear descriptor system (43). When the obtained results in Section III are applied to the mechanical systems in Section IV, the resulting closed-loop system is locally asymptotically stable. An alternative estimate of the domain of attraction is the outmost Lyapunov level contained in the domain of validity $\Omega$ of the T-S fuzzy model. This also coincides with the analysis and synthesis for a nonlinear system that usually may not provide a global solution [46][48].

\section{EXAMPLES}

In this section, three examples will be provided to show the effectiveness and superiority of the results proposed. To avoid the chattering, the term $\frac{s(t)}{\|s(t)\|}$ in the sliding mode controller will be replaced by $\frac{s(t)}{\|s(t)\|+0.001}$ for the simulation.

Example 1: Consider the problem of balancing the inverted pendulum on a cart shown in Fig. 1. The equations of motion are given by

$$
\begin{aligned}
& \left(J+m l^{2}\right) \ddot{\theta}+m l \cos (\theta) \ddot{\tilde{x}}=m g l \sin (\theta) \\
& (M+m) \ddot{\tilde{x}}+m l \ddot{\theta} \cos (\theta)=u+m l \dot{\theta}^{2} \sin (\theta)
\end{aligned}
$$

where $\theta$ is the angular displacement of the stick from the vertical position; $\tilde{x}$ is the horizontal displacement of the cart; $u$ is the control force applied to the car; $M$ and $m$ denote the mass of the car and the mass of the stick respectively; $2 l$ is the length of the stick; $J=\frac{m l^{2}}{3}$ is the moment of inertia of the stick with respect to its center of gravity; $g$ is the acceleration due to gravity;

Define $x(t)=\left[\begin{array}{lll}x_{1}(t) & x_{2}(t) x_{3}(t) x_{4}(t)\end{array}\right]^{T}, x_{1}(t)=\theta$, $x_{2}(t)=\dot{\theta}, x_{3}(t)=\tilde{x}, x_{4}(t)=\dot{\tilde{x}}$, the motion equation (46) can be represented by the following descriptor nonlinear system

$$
\begin{aligned}
& {\left[\begin{array}{cccc}
1 & 0 & 0 & 0 \\
0 & \frac{4 m l^{2}}{3} & 0 & m l \cos \left(x_{1}(t)\right) \\
0 & 0 & 1 & 0 \\
0 & m l \cos \left(x_{1}(t)\right) & 0 & M+m
\end{array}\right] \dot{x}(t) } \\
= & {\left[\begin{array}{c}
x_{2}(t) \\
m g l \sin \left(x_{1}(t)\right) \\
x_{4}(t) \\
m l x_{2}^{2}(t) \sin \left(x_{1}(t)\right)
\end{array}\right]+\left[\begin{array}{l}
0 \\
0 \\
0 \\
1
\end{array}\right] u(t) }
\end{aligned}
$$

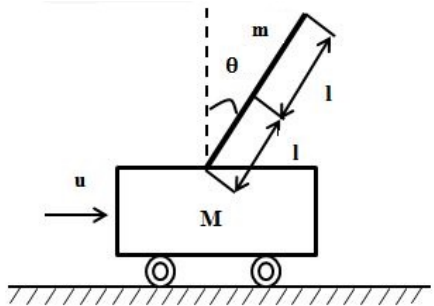

Fig. 1. A car with an inverted pendulum

To make the motion equation (46) tractable, the traditional method [2], [7] is transforms (46) into the following normal form

$$
\begin{aligned}
\dot{x}(t)= & {\left[\begin{array}{c}
x_{2}(t) \\
\frac{g \sin \left(x_{1}(t)\right)}{4 l / 3-m l a \cos ^{2}\left(x_{1}(t)\right)} \\
x_{4}(t) \\
\frac{-m a g \sin \left(2 x_{1}(t)\right) / 2}{4 / 3-m a \cos ^{2}\left(x_{1}(t)\right)}
\end{array}\right] } \\
& +\left[\begin{array}{c}
0 \\
\frac{-a \cos \left(x_{1}(t)\right)}{4 l / 3-m l a \cos ^{2}\left(x_{1}(t)\right)} \\
0 \\
4 a / 3 \\
\frac{4 a \cos ^{2}\left(x_{1}(t)\right)}{4 / 3-m a \cos ^{2}}
\end{array}\right](u(t)+f(x(t)))
\end{aligned}
$$

where $a=\frac{1}{M+m}, f(x(t))=m l x_{2}^{2}(t) \sin \left(x_{1}(t)\right)$.

Note that the coefficient matrices corresponding to the input vector $u(t)$ in the system (47) and the system (48) are $B_{1}=\left[\begin{array}{llll}0 & 0 & 0 & 1\end{array}\right]^{T}$ and $B_{2}(x(t))=$ $\left[0 \frac{-a \cos \left(x_{1}(t)\right)}{4 l / 3-m l a \cos ^{2}\left(x_{1}(t)\right)} 0 \frac{4 a / 3}{4 / 3-m a \cos ^{2}\left(x_{1}(t)\right)}\right]^{T}$, respectively. It can be seen that the inverse operation around the derivativeterm coefficient results in a nonlinear coefficient $B_{2}(x(t))$. Therefore, the nonlinear coefficient $B_{2}(x(t))$ arises purely from mathematical operations required to obtain a specific canonical form.

Due to physical limitations, it is reasonable to assume $x(t) \in \Omega:=\left\{x(t):-\xi_{1} \leq x_{1}(t) \leq \xi_{1},-\xi_{2} \leq x_{2}(t) \leq\right.$ $\left.\xi_{2},-\xi_{3} \leq x_{3}(t) \leq \xi_{3},-\xi_{4} \leq x_{4}(t) \leq \xi_{4}\right\}$, where $\xi_{1}, \xi_{2}$, $\xi_{3}$ and $\xi_{4}$ are bounded positive constants. Using the sector nonlinearity approach, the nonlinear systems (47) and (48) can be exactly represented by the corresponding T-S fuzzy system in the compact set $\Omega$. Note that there are 3 nonlinear functions $\left(\frac{1}{4 / 3-m a \cos ^{2}\left(x_{1}(t)\right)}, \sin \left(x_{1}(t)\right), \cos \left(x_{1}(t)\right)\right)$ in the nonlinear system (48). This implies that the corresponding T-S fuzzy system contains $8=2^{3}$ fuzzy rules. While 2 nonlinear functions $\left(\cos \left(x_{1}(t)\right), \sin \left(x_{1}(t)\right)\right)$ arise in the nonlinear system (47), thus, only $4=2^{2}$ fuzzy rules are required to represent the system (47).

Define $z_{1}(t)=\cos \left(x_{1}(t)\right), \quad z_{2}(t)=\sin \left(x_{1}(t)\right)$, $z_{3}(t)=m l x_{2}(t) \sin \left(x_{1}(t)\right)$. Since the maximum and minimum values of $z_{1}(t), \quad z_{2}(t)$ and $z_{3}(t)$ in the set $\Omega$ are $\max _{x_{1}(t)} z_{1}(t)=b_{1}, \min _{x_{1}(t)} z_{1}(t)=$ $b_{2}, \max _{x_{1}(t)} z_{2}(t)=c_{1} x_{1}(t), \min _{x_{1}(t)} z_{2}(t)=$ $c_{2} x_{1}(t), \max _{x_{1}(t), x_{2}(t)} z_{3}(t)=d_{1}, \min _{x_{1}(t), x_{2}(t)} z_{3}(t)=d_{2}$. 
$z_{1}(t), z_{2}(t)$ and $z_{3}(t)$ can be respectively rewritten by $z_{1}(t)=\cos \left(x_{1}(t)\right)=v_{1}\left(z_{1}(t)\right) b_{1}+v_{2}\left(z_{1}(t)\right) b_{2}, \quad z_{2}(t)=$ $\sin \left(x_{1}(t)\right)=h_{1}\left(z_{2}(t)\right) c_{1} x_{1}(t)+h_{2}\left(z_{2}(t)\right) c_{2} x_{1}(t), z_{3}(t)=$ $\frac{d_{1}+d_{2}}{2}+\alpha(t) \frac{d_{1}-d_{2}}{2}=d_{3}+\alpha(t) d_{4}$, where $\alpha(t) \in[-1,1]$ and the membership functions are calculated as $v_{1}\left(z_{1}(t)\right)=\frac{z_{1}(t)-b_{2}}{b_{1}-b_{2}}, v_{2}\left(z_{1}(t)\right)=1-v_{1}\left(z_{1}(t)\right), h_{1}\left(z_{2}(t)\right)=$ $\left\{\begin{array}{cc}\frac{z_{2}(t)-c_{2} \arcsin \left(z_{2}(t)\right)}{\left(c_{1}-c_{2}\right) \arcsin \left(z_{2}(t)\right)}, & z_{2}(t) \neq 0 \\ 1, & \text { otherwise, }\end{array}, \quad h_{2}\left(z_{2}(t)\right)=\right.$ $1-h_{1}\left(z_{2}(t)\right)$.

Therefore, the nonlinear descriptor system (47) can be exactly represented by the following T-S fuzzy descriptor system

$$
\begin{aligned}
\sum_{k=1}^{2} v_{k}(z(t)) E_{k} \dot{x}(t) & =\sum_{i=1}^{2} h_{i}(z(t)) A_{i} x(t)+B u(t) \\
y(t) & =C x(t)
\end{aligned}
$$

where $z(t)=\left[\begin{array}{c}z_{1}(t) \\ z_{2}(t)\end{array}\right], C=\left[\begin{array}{llll}1 & 0 & 0 & 0 \\ 0 & 0 & 1 & 0\end{array}\right]$, $N_{A}=\left[\begin{array}{llll}0 & 1 & 0 & 0\end{array}\right], F_{A}(t)=\alpha(t), \quad E_{i}=$ $\left[\begin{array}{cccc}1 & 0 & 0 & 0 \\ 0 & \frac{4 m l^{2}}{3} & 0 & m l b_{i} \\ 0 & 0 & 1 & 0 \\ 0 & m l b_{i} & 0 & M+m\end{array}\right], A_{i}=\left[\begin{array}{cccc}0 & 1 & 0 & 0 \\ m g l c_{i} & 0 & 0 & 0 \\ 0 & 0 & 0 & 1 \\ 0 & d_{3} & 0 & 0\end{array}\right]$, $i=1,2, B=\left[\begin{array}{l}0 \\ 0 \\ 0 \\ 1\end{array}\right], M_{A}=\left[\begin{array}{c}0 \\ 0 \\ 0 \\ d_{4}\end{array}\right]$.

For the inverted pendulum model, the parameter values are given by $m=2 \mathrm{~kg}, M=8 \mathrm{~kg}, g=9.8 \mathrm{~m} / \mathrm{s}^{2}, l=0.5 \mathrm{~m}, \xi_{1}=$ $70^{\circ} \pi / 180^{\circ} \mathrm{rad}, \xi_{2}=3 \mathrm{rad} / \mathrm{s}$. A state feedback sliding mode control strategy and an observer-based sliding mode control strategy will be developed.

Case 1: Assume that $\theta, \dot{\theta}, \tilde{x}$ and $\dot{\tilde{x}}$ are measurable. In the compact set $\Omega$, the nonlinear system (48) can be exactly represented by the corresponding T-S fuzzy system with 8 fuzzy rules. By using the method in [7], the LMIs therein are found to be infeasible. Whereas, $X_{1 k}, X_{2 k}, K_{1 k i}, K_{2 k i}$, $k, i=1,2$ can be computed in terms of Corollary 4 with $\tau=1$. By the obtained parameters, the sliding variable can be obtained as follows

$$
\begin{aligned}
s(t)= & 10 x_{4}(t)+x_{2}(t) \cos x_{1}(t)-x_{2}(0) \cos x_{1}(0) \\
& -10 x_{4}(0)+\int_{0}^{t}\left(x_{2}^{2}(\tau) \sin x_{1}(\tau)\right. \\
& -\sum_{k=1}^{2} \sum_{i=1}^{2} v_{k}(z(\tau)) h_{i}(z(\tau)) K_{1 k i} \\
& \left.\left.\times\left(\sum_{k=1}^{2} v_{k}(z(\tau))\right) X_{1 k}\right)^{-1} x(\tau)\right) d \tau
\end{aligned}
$$

Using Corollary 3, the following sliding mode controller can
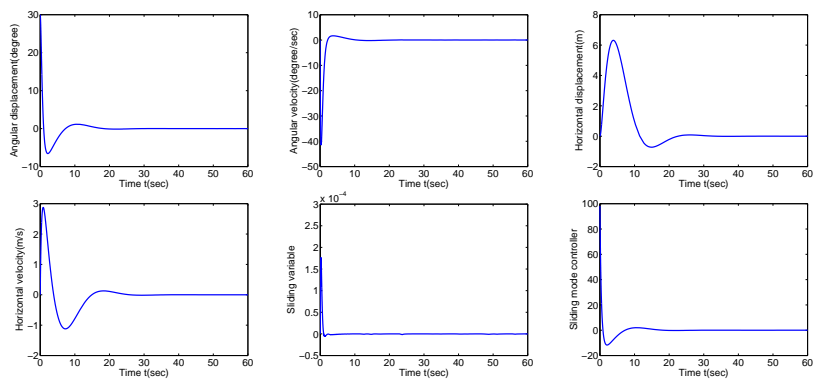

Fig. 2. Simulation results for Case 1 in Example 1 under the initial condition $x(0)=\left[\begin{array}{llll}30^{\circ} & 0 & 0 & 0\end{array}\right]^{T}$. (a)-(d) States. (e) Sliding variable. (f) Sliding mode controller.
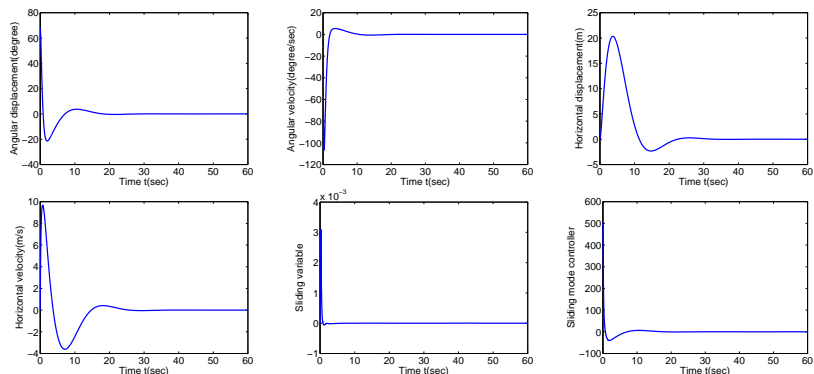

Fig. 3. Simulation results for Case 1 in Example 1 under the initial condition $x(0)=\left[\begin{array}{llll}68^{\circ} & 0 & 0 & 0\end{array}\right]^{T}$. (a)-(d) States. (e) Sliding variable. (f) Sliding mode controller.

be designed

$$
\begin{aligned}
u(t)= & \sum_{k=1}^{2} \sum_{i=1}^{2} v_{k}(z(t)) h_{i}(z(t)) K_{1 k i} \\
& \left.\times\left(\sum_{k=1}^{2} v_{k}(z(t))\right) X_{1 k}\right)^{-1} x(t) \\
& -\left(2.8191\left\|x_{2}(t)\right\|+0.001\right) \frac{s(t)}{\|s(t)\|}
\end{aligned}
$$

For different initial conditions, the time responses of the resulting closed-loop system, sliding variable and sliding mode controller are shown in Fig. 2 and Fig. 3. This shows that the resulting closed-loop system is asymptotically stable even when the initial angular displacement of the stick is $68^{\circ}$. It can be seen in Fig. 2 and Fig. 3 that compared with [7], the control forces seem somewhat larger, whereas, the maximum horizontal displacement is much smaller and the rate of convergence of the plant is faster. Moreover, the initial angular displacement of the inverted pendulum that can be stabilized by the method in this paper is much larger that that by [7]. In addition, it can be observed that with the increase of the initial angular displacements, the control forces that stabilize the inverted pendulum are also increasing, which is intuitively reasonable.

Case 2: When only the angular displacement of the stick $\theta$ and the horizontal displacement of the cart $\tilde{x}$ can be measured, by designing T-S fuzzy descriptor observer (5) and applying Theorem 2 with $\tau=1$, The gain matrices $L_{k i}, X_{1 k}, X_{2 k}$, $X_{3 i}, X_{4 i}, K_{1 k i}, K_{2 k i}, k, i=1,2$ can be obtained. With these 

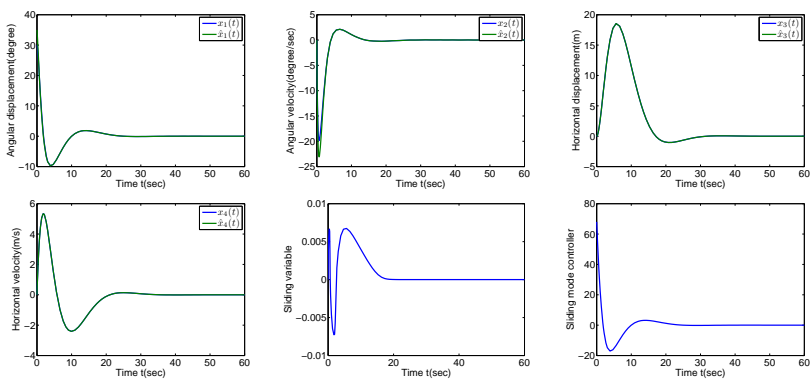

Fig. 4. Simulation results for Case 2 in Example 1 under the initial conditions $x(0)=\left[\begin{array}{llll}30^{\circ} & 0 & 0 & 0\end{array}\right]^{T}, \hat{x}(0)=\left[\begin{array}{llll}35^{\circ} & 0 & 0 & 0\end{array}\right]^{T}$. (a)-(d) States and their estimate values. (e) Sliding variable. (f) Sliding mode controller.

parameters, the observer-based sliding variable can be obtained as

$$
\begin{aligned}
& s(t)=\int_{0}^{t}\left(\dot{\hat{x}}_{2}(\tau) \cos x_{1}(\tau)+10 \dot{\hat{x}}_{4}(\tau)\right. \\
& -\sum_{k=1}^{2} \sum_{i=1}^{2} v_{k}(z(\tau)) h_{i}(z(\tau))\left[K_{1 k i} K_{2 k i}\right] \\
& \left(\sum_{k=1}^{2} \sum_{i=1}^{2} v_{k}(z(\tau)) h_{i}(z(\tau))\left[\begin{array}{cc}
X_{1 k} & X_{2 k} \\
X_{3 i} & X_{4 i}
\end{array}\right]\right)^{-1} \\
& \left.\times\left[\begin{array}{c}
\hat{x}(\tau) \\
\hat{\hat{x}}(\tau)
\end{array}\right]\right) d \tau
\end{aligned}
$$

where $\hat{x}(t)=\left[\hat{x}_{1}(t) \hat{x}_{2}(t) \hat{x}_{3}(t) \hat{x}_{4}(t)\right]^{T}$ and $\dot{\hat{x}}(t)=$ $\left[\dot{\hat{x}}_{1}(t) \dot{\hat{x}}_{2}(t) \dot{\hat{x}}_{3}(t) \dot{\hat{x}}_{4}(t)\right]^{T}$ are the states of the observer (5) with the parameters in this example.

By Theorem 1, the observer-based sliding mode controller is defined by

$$
\begin{aligned}
u(t)= & \sum_{k=1}^{2} \sum_{i=1}^{2} v_{k}(z(t)) h_{i}(z(t)) K_{k i} \\
& \left(\sum_{k=1}^{2} \sum_{i=1}^{2} v_{k}(z(t)) h_{i}(z(t)) X_{k i}\right)^{-1}\left[\begin{array}{c}
\hat{x}(t) \\
\hat{x}(t)
\end{array}\right] \\
& -\left(\| B^{T} \sum_{k=1}^{2} \sum_{i=1}^{2} v_{k}(z(t)) h_{i}(z(t)) L_{k i}\right. \\
& \times(y(t)-C \hat{x}(t)) \|+0.001) \frac{s(t)}{\|s(t)\|}
\end{aligned}
$$

For different initial conditions, the time responses of the resulting closed-loop system, the observer system, the sliding variable and the sliding mode controller are depicted in Fig. 4 and Fig. 5. This shows that the resulting closed-loop system is asymptotically stable and the states of the observer system can track those of the original system asymptotically even when the initial conditions of the original system and observer system are different. It is noted that when only partial state information is available, the methods in [7], [8], [11] cannot be adopted because they are only applicable to state-based sliding mode control problems.

Since the sector nonlinearity approach is employed in this paper, the T-S fuzzy descriptor system can exactly represent the nonlinear descriptor system in a compact set of the state
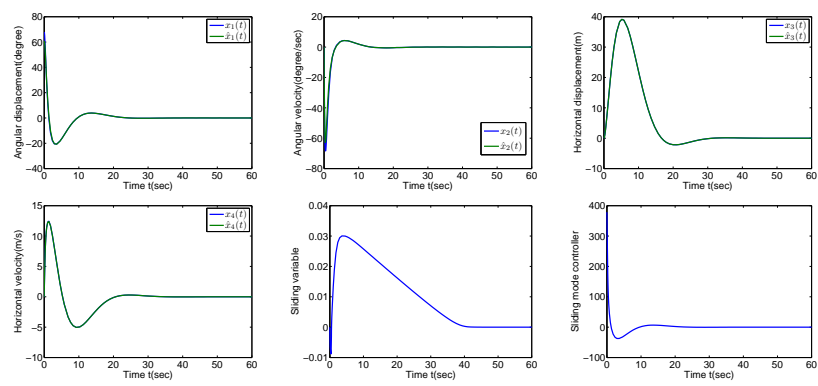

Fig. 5. Simulation results for Case 2 in Example 1 under the initial conditions $x(0)=\left[\begin{array}{llll}68^{\circ} & 0 & 0 & 0\end{array}\right]^{T}, \hat{x}(0)=\left[\begin{array}{llll}60^{\circ} & 0 & 0 & 0\end{array}\right]^{T}$. (a)-(d) States and their estimate values. (e) Sliding variable. (f) Sliding mode controller.

space including the origin. Then if the LMIs in Theorem 2 or Corollary 4 are solvable, the proposed sliding mode control strategy can stabilize the nonlinear descriptor system in such a compact set. This example is provided as an academic study to illustrate the obtained results, how to apply the results in this paper to practical applications needs the further research.

Example 2: Consider the nonlinear system

$$
(1+a \cos \theta(t)) \ddot{\theta}(t)=-b \dot{\theta}^{3}(t)+c \theta(t)+d u(t)
$$

Introduce the state vector $x(t)=\left[x_{1}(t) x_{2}(t) x_{3}(t)\right]^{T}$ with $x_{1}(t)=\theta(t), x_{2}(t)=\dot{\theta}(t)$ and $x_{3}(t)=\ddot{\theta}(t)$, [16] describes the nonlinear system (54) by the following descriptor model

$$
E \dot{x}(t)=A(x(t)) x(t)+B u(t)
$$

$$
\begin{gathered}
\text { where } \quad E \\
\left.\begin{array}{ccc}
0 & 1 & 0 \\
0 & 0 & 1 \\
c & -b x_{2}^{2} & -1-a \cos x_{1}(t)
\end{array}\right], B=\left[\begin{array}{lll}
1 & 0 & 0 \\
0 & 1 & 0 \\
0 & 0 & 0
\end{array}\right], A(x(t) \\
{\left[\begin{array}{l}
0 \\
d
\end{array}\right] .}
\end{gathered}
$$

When the variables $\theta, \dot{\theta}$ and $\ddot{\theta}$ are available, [16], [31] stabilize the descriptor model with time-delay by state feedback controller and state feedback sliding mode controller respectively. Whereas, from the nonlinear system, it is observed that the available variable may be only $\theta$. Since the variable $\dot{\theta}$ is unavailable, the descriptor system (55) can not be represented by the T-S fuzzy descriptor system in [16], [31] and the state-based controllers [16], [31] are invalid. Assume that $\theta, \dot{\theta}$ and $\ddot{\theta}$ takes values in the compact set $\Upsilon=\left\{-\phi_{1} \leq \theta \leq \phi_{1},-\phi_{2} \leq \dot{\theta} \leq \phi_{2},-\phi_{3} \leq \ddot{\theta} \leq \phi_{3}\right\}$ where $\phi_{1}, \phi_{2}$ and $\phi_{3}$ are bounded positive constants. Using the sector nonlinearity approach [1], the descriptor model (55) can be exactly represented in the compact set $\Upsilon$ by the following T-S fuzzy descriptor system

$$
\begin{aligned}
E \dot{x}(t) & =\sum_{i=1}^{2} h_{i}\left(x_{1}(t)\right)\left(\left(A_{i}+\Delta A\right) x(t)+B u(t)\right) \\
y(t) & =C x(t)
\end{aligned}
$$

where $A_{1}=\left[\begin{array}{ccc}0 & 1 & 0 \\ 0 & 0 & 1 \\ c & -b \phi_{2}^{2} / 2 & -1-a\end{array}\right], A_{2}=$
$\left[\begin{array}{ccc}0 & 1 & 0 \\ 0 & 0 & 1 \\ c & -b \phi_{2}^{2} / 2 & -1-a \cos \phi_{1}\end{array}\right], M_{1}=\left[\begin{array}{c}0 \\ 0 \\ b \phi_{2}^{2} / 2\end{array}\right], \Delta A=$ 

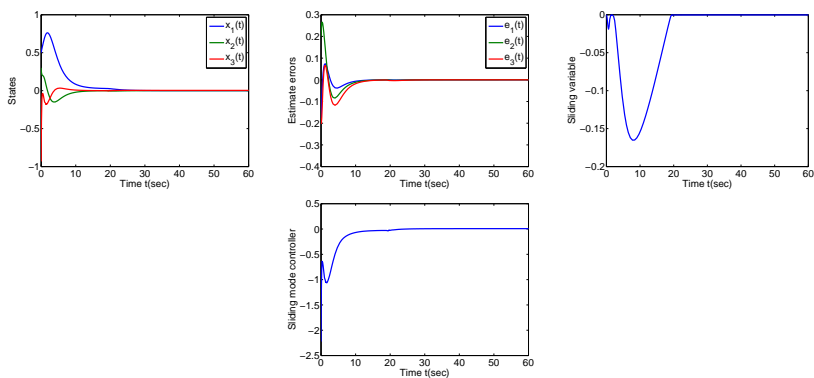

Fig. 6. Simulation results in Example 2 under the initial conditions $x(0)=$ $\left[\begin{array}{lll}0.5 & 0.3 & 0\end{array}\right]^{T}, \hat{x}(0)=\left[\begin{array}{llll}0.7 & 0.1 & 0\end{array}\right]^{T}$. (a) States. (b) Estimate errors. (c) Sliding variable. (d) Sliding mode controller.

$M_{1} F_{1}(t) N_{1}, N_{1}=\left[\begin{array}{lll}0 & 1 & 0\end{array}\right], C=\left[\begin{array}{lll}1 & 0 & 0\end{array}\right],-1 \leq F_{1}(t) \leq$ $1, h_{1}\left(x_{1}(t)\right)=\frac{\cos x_{1}(t)-\cos \phi_{1}}{1-\cos \phi_{1}}, h_{2}\left(x_{1}(t)\right)=1-h_{1}\left(x_{1}(t)\right)$.

For the purpose of simulation, it is assumed that $a=b=$ $c=d=1, \phi_{1}=\pi / 3, \phi_{2}=2$. By checking the LMIs in Corollaries 1 and 2, it is found that only the LMIs in Corollary 1 are feasible. The observer gains and controller gains are

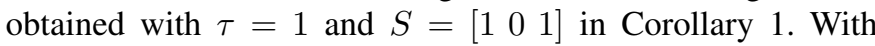
the above parameters, the following sliding variable can be constructed

$$
\begin{aligned}
s(t)= & \hat{x}_{1}(t)-\hat{x}_{1}(0) \\
& +\int_{0}^{t}\left(h_{1}\left(x_{1}(\tau)\right)\left[\begin{array}{lll}
0.8564 & 19.4584 & 1.7661
\end{array}\right]\right. \\
& \left.+h_{2}\left(x_{1}(\tau)\right)\left[\begin{array}{lll}
1.6833 & 28.7538 & 1.3580
\end{array}\right]\right) \hat{x}(\tau) d \tau
\end{aligned}
$$

where $\hat{x}(t)=\left[\hat{x}_{1}(t) \hat{x}_{2}(t) \hat{x}_{3}(t)\right]^{T}$ is the states of the observer (5) with the parameters herein.

By Theorem 1, the observer-based sliding mode controller can be designed as

$$
\begin{aligned}
u(t)= & \left(h_{1}\left(x_{1}(t)\right)[-1.8564-18.45840 .2339]\right. \\
& \left.+h_{2}\left(x_{1}(t)\right)[-2.6833-27.75380 .1420]\right) \hat{x}(t) \\
& \left.-\left(0.5312 \| x_{1}(t)-\hat{x}_{1}(t)\right) \|+0.02\right) \frac{s(t)}{\|s(t)\|}
\end{aligned}
$$

The time responses of the resulting closed-loop system, the error system, the sliding variable and the sliding mode controller are shown in Fig. 6. It shows that the resulting closedloop system is asymptotically stable and the error system is convergent to zero asymptotically. The control strategies in [16], [19], [31] are based on the state feedback and are not applicable when only output information is available.

Example 3: Consider the following T-S fuzzy descriptor system

$$
\begin{aligned}
E \dot{x}(t) & =\sum_{i=1}^{2} h_{i}\left(A_{i}+\Delta A\right) x(t)+B u(t) \\
y(t) & =C x(t)
\end{aligned}
$$

where $x(t)=\left[x_{1}(t) x_{2}(t) x_{3}(t)\right]^{T}$, the membership functions are $h_{1}\left(x_{1}(t)\right)=\cos \left(x_{1}(t)\right), h_{2}\left(x_{1}(t)\right)=1-$ $\cos \left(x_{1}(t)\right),\left|x_{1}(t)\right| \leq \frac{\pi}{3}$, and the parameters are given by $E=\left[\begin{array}{lll}1 & 0 & 0 \\ 0 & 1 & 0 \\ 0 & 0 & 0\end{array}\right], A_{1}=\left[\begin{array}{ccc}1.5 & 0 & 0 \\ 1 & 1 & 0.1 \\ 1 & 0.5 & -1\end{array}\right], A_{2}=$
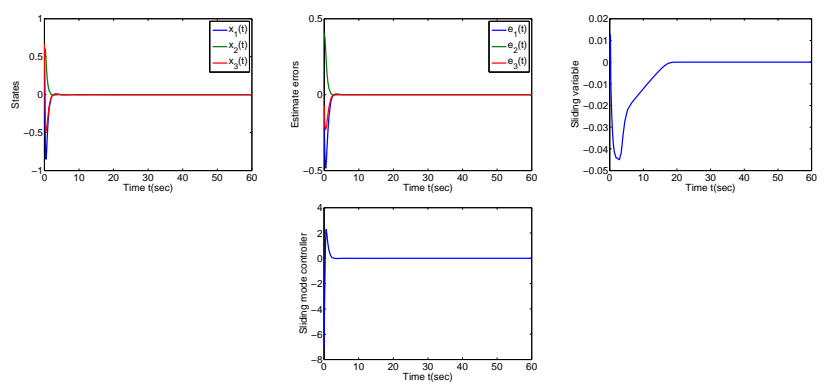

Fig. 7. Simulation results in Example 3 under the initial conditions $x(0)=$ $\left[\begin{array}{lll}0.5 & 0.5 & 0\end{array}\right]^{T}, \hat{x}(0)=\left[\begin{array}{lll}0.7 & 0.1 & 0\end{array}\right]^{T}$. (a) States. (b) Estimate errors. (c) Sliding variable. (d) Sliding mode controller.

$$
\begin{aligned}
& {\left[\begin{array}{ccc}
2.5 & 0 & 0 \\
1.5 & -1 & 0 \\
1 & 0.3 & -2
\end{array}\right], B=\left[\begin{array}{l}
1 \\
0 \\
0
\end{array}\right], M_{1}=\left[\begin{array}{l}
0.2 \\
0.3 \\
0.2
\end{array}\right],} \\
& C=\left[\begin{array}{ccc}
1 & 0 & 0 \\
0 & 1 & 0
\end{array}\right], \Delta A=M_{1} \sin \left(x_{1}(t)\right) N_{1}, N_{1}= \\
& {\left[\begin{array}{lll}
0.1 & 0.2 & 0.1
\end{array}\right] .}
\end{aligned}
$$

By checking the LMIs in Corollaries 1 and 2, it is found that both of them are feasible. Without loss of generality, the observer gains and controller gains by Corollary 2 with $\tau=$ 0.1 are used. With the above parameters, the observer-based sliding variable is constructed by

$$
\begin{aligned}
s(t)= & \hat{x}_{1}(t)-\hat{x}_{1}(0) \\
& -\int_{0}^{t}\left(\cos x_{1}(\tau)\left[\begin{array}{lll}
27.6402 & 70.1864 & -8.2461
\end{array}\right]\right. \\
& \left.+\left[\begin{array}{lll}
-51.5620 & -130.3643 & 12.8378
\end{array}\right]\right) \hat{x}(\tau) d \tau
\end{aligned}
$$

where $\hat{x}(t)=\left[\hat{x}_{1}(t) \hat{x}_{2}(t) \hat{x}_{3}(t)\right]^{T}$ is the states of the observer (5) with the obtained parameters.

By Theorem 1, the observer-based sliding mode controller can be designed as follows

$$
\begin{aligned}
u(t)= & \left(\cos x_{1}(t)[28.640270 .1864-8.2461]\right. \\
& +[-54.0620-130.364312 .8378]) \hat{x}(t) \\
& -\left(0.002+\|\left(\cos x_{1}(t)\left[\begin{array}{ll}
3.3345 & 4.6251
\end{array}\right]\right.\right. \\
& +[8.12729 .8669])(y(t)-C \hat{x}(t)) \|) \frac{s(t)}{\|s(t)\|}
\end{aligned}
$$

The time responses of the resulting closed-loop system, the error system, the sliding variable and the sliding mode controller are shown in Fig. 7. It is seen that the resulting closed-loop system is asymptotically stable and the state trajectories of error system asymptotically converges to zero. Therefore, the single-step design approach in Theorem 2 is effective to deal with the observer-based sliding mode control problem for T-S fuzzy descriptor system.

\section{Conclusions}

This paper has studied observer-based fuzzy control of nonlinear descriptor systems via integral sliding modes. By virtue of the T-S fuzzy descriptor system, the observer-based sliding mode control strategy for nonlinear descriptor systems was presented in terms of LMIs. The two-step approach and the single-step approach were respectively used to determine 
the observer gains and coefficients in the switching function. Application of the proposed integral sliding mode control to a class of mechanical systems was investigated and an alternative approach to the sliding mode control of mechanical systems in the form of nonlinear descriptor systems provided. It was shown that with the descriptor system representation, different input matrices of the resulting T-S system were not artificially introduced and the number of fuzzy rules for the corresponding T-S fuzzy system was reduced.

\section{ACKNOWLEDGMENT}

The authors would like to express sincere thanks to the Associate Editor and reviewers for their kind help and valuable comments which improved the quality of this paper.

\section{REFERENCES}

[1] K. Tanaka and H.O. Wang, Fuzzy Control Systems Design and Analysis: A Linear Matrix Inequality Approach. New York, USA: John Wiley \& Sons, 2001.

[2] M.C.M. Teixeira and S.H. Żak , "Stabilizing controller design for uncertain nonlinear systems using fuzzy models," IEEE Trans. Fuzzy Syst., vol. 7, no. 2, pp. 133-142, Apr. 1999.

[3] X. Liu and Q. Zhang, "New approaches to $H_{\infty}$ controller designs based on fuzzy observers for T-S fuzzy systems via LMI," Automatica, vol. 39, no. 9 , pp. 1571-1582, Sep. 2003

[4] C. Lin, Q.-G. Wang, and T.H. Lee, "Stabilization of uncertain fuzzy timedelay systems via variable structure control approach," IEEE Trans. Fuzzy Syst., vol. 13, no. 6, pp. 787-798, Dec. 2005

[5] C. Lin, Q.-G. Wang, and T.H. Lee, "Improvement on observer-based $H_{\infty}$ control for T-S fuzzy systems," Automatica, vol. 41, no. 9, pp. 1651-1656, Sep. 2005.

[6] G. Feng, "A survey on analysis and design of model-based fuzzy control systems," IEEE Trans. Fuzzy Syst., vol. 14, no. 5, pp. 676-697, Oct. 2006.

[7] H.H. Choi, "Robust stabilization of uncertain fuzzy systems using variable structure system approach," IEEE Trans. Fuzzy Syst., vol. 16, no. 3, pp. 715-724, Jun. 2008.

[8] Z. Xi, G. Feng, and T. Hesketh, "Piecewise integral sliding-mode control for T-S fuzzy systems," IEEE Trans. Fuzzy Syst., vol. 19. no. 1, pp. 65-74, Feb. 2011.

[9] H. Li, J. Yu, C. Hilton, and H. Liu, "Adaptive sliding-mode control for nonlinear active suspension vehicle systems using T-S fuzzy approach," IEEE Trans. Ind. Electron., vol. 60, no. 8, pp. 3328-3338, May. 2013.

[10] M.H. Asemani and V.J. Majd, "A robust $H_{\infty}$ observer-based controller design for uncertain T-S fuzzy systems with unknown premise variables via LMI," Fuzzy Set Syst., vol. 212, pp. 21-40, Feb. 2013.

[11] Q. Gao, L. Liu, G. Feng, Y. Wang, and J. Qiu, "Universal fuzzy integral sliding-mode controllers based on T-S fuzzy models," IEEE Trans. Fuzzy Syst., vol. 22, no. 2, pp. 350-362, Apr. 2014.

[12] S. Wen, M.Z.Q. Chen, Z. Zeng, X. Yu, and T. Huang, "Fuzzy control for uncertain vehicle active suspension systems via dynamic sliding-mode approach," IEEE Trans. Syst. Man Cybern.: Syst., vol. 47, no. 1, pp. 2432, May. 2017.

[13] T. Taniguchi, K. Tanaka, K. Yamafuji, and H.O. Wang, "Fuzzy descriptor systems: stability analysis and design via LMIs," in Pro. 1999 Amer. Control Conf., vol. 3, San Diego, California USA, 1999, pp. 1827-1831.

[14] T. Taniguchi, K. Tanaka, and H.O. Wang, "Fuzzy descriptor systems and nonlinear model following control," IEEE Trans. Fuzzy Syst., vol. 8, no. 4, pp. 442-452, Aug. 2000.

[15] C.-P. Huang, "Stability analysis of discrete singular fuzzy systems," Fuzzy Sets Syst., vol. 151, no. 1, pp. 155-165, Apr. 2005.

[16] C. Lin, Q.-G. Wang, and T.H. Lee, "Stability and stabilization of a class of fuzzy time-delay descriptor systems," IEEE Trans. Fuzzy Syst., vol. 14, no. 4, pp. 542-551, Aug. 2006.

[17] S. Xu, B. Song, J. Lu, and J. Lam, "Robust stability of uncertain discretetime singular fuzzy systems," Fuzzy Sets Syst., vol. 158, no. 20, pp. 23062316, Oct. 2007.

[18] H. Zhang, Y. Shen, G. Feng, "Delay-dependent stability and $H_{\infty}$ control for a class of fuzzy descriptor systems with time-delay," Fuzzy Sets Syst., vol. 160. no. 12, pp. 1689-1707, Jun. 2009.
[19] C. Han, G. Zhang, L. Wu, and Q. Zeng, "Sliding mode control of T-S fuzzy descriptor systems with time-delay," J. Franklin Inst., vol. 349, no. 4, pp. 1430-1444, May. 2012.

[20] V. Estrada-Manzo, T.M. Guerra, Z. Lendek, and M. Bernal, "Improvements on non-quadratic stabilization of continuous-time Takagi-Sugeno descriptor models," in Proc. IEEE Int. Conf. Fuzzy Syst., Hyderabad, India, 2013, pp. 1-6.

[21] F. Li, P. Shi, L. Wu, and X. Zhang, "Fuzzy-model-based D-stability and nonfragile control for discrete-time descriptor systems with multiple delays," IEEE Trans. Fuzzy Syst., vol. 22, no. 4, pp. 1019-1025, Aug. 2014.

[22] M. Chadli, H.R. Karimi, and P. Shi, "On stability and stabilization of singular uncertain Takagi-Sugeno fuzzy systems," J. Franklin Inst., vol. 351, no. 3, pp. 1453-1463, Mar. 2014.

[23] V. Estrada-Manzo, Z. Lendek, T.M. Guerra, and P. Pudlo, "Controller design for discrete-time descriptor models: a systematic LMI approach," IEEE Trans. Fuzzy Syst., vol. 23, no. 5, pp. 1608-1621, Oct. 2015.

[24] P. Liu, T.-W. Yang, and C.-E. Yang, "Robust observer-based output feedback control for fuzzy descriptor systems," Expert Syst. Appl., vol. 40, no. 11, pp. 4503-4510, Sep. 2013.

[25] H. Gassara, A. El Hajjaji, M. Kchaou, and M. Chaabane, "Observer based $(Q, V, R)$ - $\alpha$-dissipative control for TS fuzzy descriptor systems with time delay," J. Franklin Inst., vol. 351, no. 1, pp. 187-206, Jan. 2014.

[26] C. Han, L. Wu, H.K. Lam , and Q. Zeng, "Nonfragile control with guaranteed cost of T-S fuzzy singular systems based on parallel distributed compensation," IEEE Trans. Fuzzy Syst., vol. 22, no. 5, pp. 1183-1196, Oct. 2014.

[27] L. Li, Q. Zhang, and B. Zhu, "Fuzzy stochastic optimal guaranteed cost control of bio-economic singular Markovian jump systems," IEEE Trans. Cybern., vol. 45, no. 11, pp. 2512-2521, Nov. 2015.

[28] J. Yoneyama and A. Ichikawa, " $H_{\infty}$-control for Takagi-Sugeno fuzzy descriptor systems," in Proc. IEEE Conf. Syst. Man Cybern., vol. 3, Tokyo, Japan, 1999, pp. 28-33.

[29] C. Yang and Q. Zhang, "Multiobjective control for T-S fuzzy singularly perturbed systems," IEEE Trans. Fuzzy Syst., vol. 17, no. 1, pp. 104-115, Feb. 2009.

[30] Q. Zhang, L. Qiao, B. Zhu, and H. Zhang, "Dissipativity analysis and synthesis for a class of T-S fuzzy descriptor systems," IEEE Trans.Syst. Man Cybern.: Syst., vol. 47, no. 8, pp. 1774-1784, Aug. 2017.

[31] M. Kchaou, H. Gassara, A. El-Hajjaji, and A. Toumi, "Dissipativitybased integral sliding-mode control for a class of Takagi-Sugeno fuzzy singular systems with time-varying delay," IET Control Theory Appl., vol. 8, no. 17, pp. 2045-2054, Nov. 2014.

[32] Q. Jia, W. Chen, Y. Zhang, and H. Li, "Fault reconstruction and faulttolerant control via learning observers in Takagi-Sugeno fuzzy descriptor systems with time delays," IEEE Trans. Industrial Electronics, vol. 62, no. 6, pp. 3885-3895, Jun. 2015.

[33] C. Edwards and S.K. Spurgeon, Sliding Mode Control: Theory and Applications. London, UK: Taylor \& Francis, 1998.

[34] L. Fridman, A. Poznyak, R. Bejarano, and J. Francisco, Robust Output LQ Optimal Control via Integral Sliding Modes. Basel, Switzerland: Birkhäuser, 2014.

[35] V. Utkin, J. Guldner, and J. Shi, Sliding Mode Control in ElectroMechanical Systems, Second Edition. London, UK: CRC Press, 2009.

[36] J. Davila, L. Fridman, and A. Levant, "Second-order sliding-mode observer for mechanical systems," IEEE Trans. Autom. Control, vol. 50, no. 11, pp. 1785-1789, May. 2005.

[37] X.-G. Yan, C. Edwards, and S.K. Spurgeon, "Output feedback sliding mode control for non-minimum phase systems with non-linear disturbances," Int. J. Control, vol. 77, no. 15, pp. 1353-1361, Oct. 2004.

[38] X.-G. Yan, S.K. Spurgeon, and C. Edwards, "State and parameter estimation for nonlinear delay systems using sliding mode techniques," IEEE Trans. Autom. Control, vol. 58, no. 4, pp. 1023-1029, Apr. 2013.

[39] X.-G. Yan, S.K. Spurgeon, and C. Edwards, "Memoryless static output feedback sliding mode control for nonlinear systems with delayed disturbances," IEEE Trans. Autom. Control, vol. 59, no. 7, pp. 1906-1912, Jul. 2014.

[40] S.K. Spurgeon, "Sliding mode observers: a survey," Int. J. Syst. Sci., vol. 39, no. 8, pp. 751-764, Aug. 2008.

[41] X.-G. Yan, S.K. Spurgeon, and C. Edwards, "Sliding mode control for time-varying delayed systems based on a reduced-order observer," Automatica, vol. 46, no. 8, pp. 1354-1362, Aug. 2010.

[42] P. Gahinet and P. Apkarian, "A linear matrix inequality approach to $H_{\infty}$ control," Int. J. Robust Nonlinear Control, vol. 4, no. 4, pp. 421-448, 1994. 
[43] K. Guelton, S. Delprat, and T.M. Guerra, "An alternative to inverse dynamics joint torques estimation in human stance based on a TakagiSugeno unknown-inputs observer in the descriptor form," Control Eng. Practice, vol. 16, no. 12, pp. 1414-1426, Dec. 2008.

[44] B. Allouche, L. Vermeiren, A. Dequidt, and M. Dambrine, "Robust control of two-wheeled self-balanced transporter on sloping ground: a Takagi-Sugeno descriptor approach," in Proc. Inter. Conf. Syst. Control, Algiers, Algeria, 2013, pp. 29-31.

[45] L. Vermeirena, A. Dequidta, M. Afrounb, and T.M. Guerra, "Motion control of planar parallel robot using the fuzzy descriptor system approach," ISA Trans., vol. 51, no. 5, pp. 596-608, Sep. 2012.

[46] T.M. Guerra, M. Bernal, K. Guelton, and S. Labiod, "Non-quadratic local stabilization for continuous-time Takagi-Sugeno models," Fuzzy Sets Syst., vol. 201, pp. 40-54, 2012.

[47] M. Klug, E.B. Castelan, V.J.S. Leite, and L.F.P. Silva, "Fuzzy dynamic output feedback control through nonlinear Takagi-Sugeno models," Fuzzy Sets Syst., vol. 263, pp. 92-111, 2015.

[48] H. Khalil, Nonlinear Systems, Third Edition. New Jersey, USA: Prentice Hall, 2002.

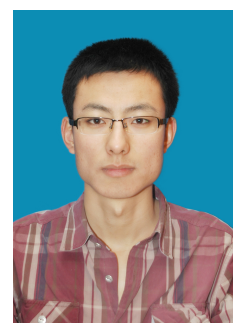

Jinghao Li received a B.Sc. degree in Mathematics from Northeastern University, China, in 2011, and his M.Sc. degree in Systems Theory from Northeastern University, China, in 2013. He is now pursuing the Ph.D degree in Control Theory and Control Engineering at Northeastern University, China. His current research interests focus on sliding mode control, descriptor system and T-S fuzzy system.

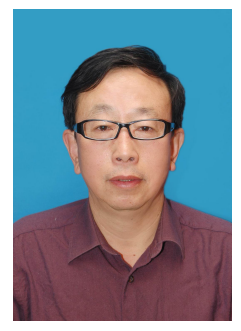

Qingling Zhang obtained B.Sc. and M.Sc. degrees in Mathematics Department, and $\mathrm{PhD}$ degree in Automatic Control Department from Northeastern University, Shenyang, China, in 1982, 1986 and 1995, respectively. He finished his two-year Postdoctoral work in Automatic Control Department of Northwestern Polytechnical University, Xian, China, in 1997. Since then he has been a Professor and has served the College of Science at Northeastern University as dean from 1997 to 2006. He was also a member of the University Teaching Advisory Committee of National Ministry of Education, and now is vice chairman of the Chinese Biomathematics Association, a member of technical committee on control theory of the Chinese Association of Automation, a member of the Chinese Association of Mathematics and Chairman of Mathematics Association of Liaoning Province. He has published 16 books and more than 600 papers on control theory and its applications. Prof. Zhang has received 14 prizes from central and local governments for his research. He has also received a Gelden Scholarship from Australia in 2000. He visited Hong Kong University, Seoul University, Alberta University, Lakehead University, Sydney University, Western Australia University, Windsor University, Hongkong Polytechnic University and the University of Kent as a Research Associate, Research Fellow, Senior Research Fellow and Visiting Professor, respectively.
Xing-Gang Yan received his B.Sc. degree in Applied Mathematics from Shaanxi Normal University, in 1985, a M.Sc. degree in Control and Optimisation from Qufu Normal University in 1991, and his Ph.D. degree in Control Engineering from Northeastern University, P. R. China in 1997. Currently, he is a Senior Lecturer at the University of Kent, United Kingdom. He was a Lecturer in Qingdao University, P. R. China from 1991 to 1994 . He has worked as a Research Fellow or Research Associate in the Northwestern Polytechnical University, China, the University of Hong Kong, China, Nanyang Technological University, Singapore and the University of Leicester, UK. He is the Editor-In-Chief of the International Journal of Engineering Research and Science \& Technology, and serves as a member of the Editorial Board for several engineering journals. His research interests include sliding mode control, decentralised control, fault detection and isolation, nonlinear control and time delay systems with applications. He has published three books, several book chapters and over 150 refereed papers.

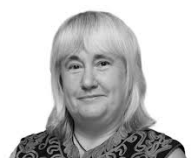

Sarah Spurgeon OBE, FREng, FInstMC, FIET, FIMA (SM'04) received B.Sc. and D.Phil. degrees from the University of York, York, U.K., in 1985 and 1988, respectively. She is currently Professor of Control Engineering and Head of Department of Electronic and Electrical Engineering at University College London in the UK. She is President of the Institute of Measurement and Control. Sarah Spurgeon's research interests are in the area of systems modelling and analysis, robust control and estimation in which areas she has published over 270 refereed research papers. She was awarded the Honeywell International Medal for 'distinguished contribution as a control and measurement technologist to developing the theory of control' in 2010 and an IEEE Millennium Medal in 2000. She is currently a member of the Council of the International Federation of Automatic Control (IFAC) and a member of the General Assembly of the European Control Association. 